\title{
Scattering of Solitons for Schrödinger Equation Coupled to a Particle
}

\author{
Alexander Komech ${ }^{1}$ \\ Faculty of Mathematics of Vienna University \\ Nordbergstrasse 15, 1090 Vienna, Austria \\ email: komech@mathematik.tu-muenchen.de \\ Elena Kopylova ${ }^{2}$ \\ M.V.Keldysh Institute of Applied Mathematics RAS \\ Miusskaya sq. 4, 125047 Moscow, Russia \\ email: ek@vpti.vladimir.ru
}

\begin{abstract}
We establish soliton-like asymptotics for finite energy solutions to the Schrödinger equation coupled to a nonrelativistic classical particle. Any solution with initial state close to the solitary manifold, converges to a sum of traveling wave and outgoing free wave. The convergence holds in global energy norm. The proof uses spectral theory and the symplectic projection onto solitary manifold in the Hilbert phase space.
\end{abstract}

\footnotetext{
${ }^{1}$ On leave Department Mechanics and Mathematics of Moscow State University. Supported partly by Max-Planck Institute of Mathematics in the Sciences (Leipzig), and Wolfgang Pauli Institute of Vienna University. 


\section{Introduction}

We continue the study of coupled systems of wave fields and particles. In [10] the KleinGordon equation coupled to a relativistic particle has been considered. Here we extend the result to the Schrödinger equation coupled to a nonrelativistic particle. We prove the long time convergence to the sum of a soliton and dispersive wave. The convergence holds in global energy norm for finite energy solution with initial state close to the solitary manifold.

We consider the Schrödinger wave function $\psi(x)$ in $\mathbb{R}^{3}$, coupled to a nonrelativistic particle with position $q$ and momentum $p$, governed by

$$
\left\{\begin{array}{l}
i \dot{\psi}(x, t)=-\Delta \psi(x, t)+m^{2} \psi(x, t)+\rho(x-q(t)) \\
\ddot{q}(t)=\frac{1}{2} \int[\bar{\psi}(x, t) \nabla \rho(x-q(t))+\psi(x, t) \nabla \bar{\rho}(x-q(t))] d x,
\end{array} \mid x \in \mathbb{R}^{3}\right.
$$

where $m>0$. Denote $\psi_{1}=\operatorname{Re} \psi, \psi_{2}=\operatorname{Im} \psi, \rho_{1}=\operatorname{Re} \rho, \rho_{2}=\operatorname{Im} \rho$.

Then the system (1.1) becomes

$$
\left\{\begin{array}{l}
\dot{\psi}_{1}(x, t)=-\Delta \psi_{2}(x, t)+m^{2} \psi_{2}(x, t)+\rho_{2}(x-q(t)), \\
\dot{\psi}_{2}(x, t)=\Delta \psi_{1}(x, t)-m^{2} \psi_{1}(x, t)-\rho_{1}(x-q(t)), \\
\ddot{q}(t)=\int\left(\psi_{1}(x, t) \nabla \rho_{1}(x-q(t))+\psi_{2}(x, t) \nabla \rho_{2}(x-q(t))\right) d x .
\end{array} \mid x \in \mathbb{R}^{3}\right.
$$

This is a Hamilton system with the Hamilton functional

$$
\begin{array}{r}
\mathcal{H}\left(\psi_{1}, \psi_{2}, q, \dot{q}\right)=\frac{1}{2} \int\left(\left|\nabla \psi_{1}(x)\right|^{2}+\left|\nabla \psi_{2}(x)\right|^{2}+m^{2}\left|\psi_{1}(x)\right|^{2}+m^{2}\left|\psi_{2}(x)\right|^{2}\right) d x \\
+\int\left(\psi_{1}(x) \rho_{1}(x-q)+\psi_{2}(x) \rho_{2}(x-q)\right) d x+\frac{1}{2}|\dot{q}|^{2} .
\end{array}
$$

We consider the Cauchy problem for the Hamilton system (1.2) which we write as

$$
\dot{Y}(t)=F(Y(t)), \quad t \in \mathbb{R} ; \quad Y(0)=Y_{0} .
$$

Here $Y(t)=\left(\psi_{1}(t), \psi_{2}(t), q(t), p(t)\right), p(t):=\dot{q}(t), Y_{0}=\left(\psi_{01}, \psi_{02}, q_{0}, p_{0}\right)$, and all derivatives are understood in the sense of distributions. Below we always deal with column vectors but often write them as row vectors. The system (1.2) is translation-invariant and admits soliton solutions

$$
Y_{a, v}(t)=\left(\psi_{v 1}(x-v t-a), \psi_{v 2}(x-v t-a), v t+a, v\right)
$$

for all $a, v \in \mathbb{R}^{3}$ with $|v|<2 m$. The states $S_{a, v}:=Y_{a, v}(0)$ form the solitary manifold

$$
\mathcal{S}:=\left\{S_{a, v}: a, v \in \mathbb{R}^{3},|v|<2 m\right\} .
$$

Our main result is the soliton asymptotics of type

$$
\psi(x, t) \sim \psi_{v_{ \pm}}\left(x-v_{ \pm} t-a_{ \pm}\right)+W_{0}(t) \psi_{ \pm}, \quad t \rightarrow \pm \infty
$$

for solutions to $(1.1)$ with initial data close to the solitary manifold $\mathcal{S}$. Here $\psi_{v_{ \pm}}=$ $\psi_{v_{ \pm} 1}+i \psi_{v_{ \pm} 2}, W_{0}(t)$ is the dynamical group of the free Schrödinger equation, $\boldsymbol{\psi}_{ \pm}$are the corresponding asymptotic scattering states, and the asymptotics hold in the global energy norm, i.e. in the norm of the Sobolev space $H^{1}\left(\mathbb{R}^{3}\right)$. For the particle trajectory we prove that

$$
\dot{q}(t) \rightarrow v_{ \pm}, \quad q(t) \sim v_{ \pm} t+a_{ \pm}, \quad t \rightarrow \pm \infty
$$


The results are established under the following conditions on the complex valued charge distributions $\rho$ :

$$
(1+|x|)^{\beta} \rho, \quad(1+|x|)^{\beta} \nabla \rho, \quad(1+|x|)^{\beta} \nabla \nabla \rho \in L^{2}\left(\mathbb{R}^{3}\right),
$$

with some $\beta>3 / 2$. We require that all "modes" of the wave field are coupled to the particle, this is formalized by the Wiener condition

$$
\hat{\rho}(k)=(2 \pi)^{-3 / 2} \int e^{i k x} \rho(x) d x \neq 0 \text { for all } k \in \mathbb{R}^{3} .
$$

It is an analogue of the Fermi Golden Rule: the coupling term $\rho(x-q)$ is not orthogonal to the eigenfunctions $e^{i k x}$ of the continuous spectrum of the linear part of the equation (cf. $[4,21,22,23]$ ).

Similar results were proved for the first time by Buslaev and Perelman $[2,3]$ for $1 \mathrm{D}$ translation invariant Schrödinger equation, and extended by Cuccagna [6] for $\mathrm{nD}$ case, $n \geq 3$. In [10] the Klein-Gordon equation coupled to a particle, is considered.

For the proofs of the asymptotics (1.7) and (1.8), we develop the approach [10] based on the Buslaev and Perelman methods $[2,3]$ : the symplectic orthogonal decomposition of the dynamics near the solitary manifold, the time decay for the linearized equation, etc. Our problem differs from [10] in the following aspects:

i) Speed of propagation for the Schrödinger equation is infinite, and the solitons exist only for the velocities $|v|<2 m$.

ii) We consider nonspherically symmetric coupled function $\rho(x)$. In this case we need additional arguments for the absence of embedded eigenvalues in the continuous spectrum.

iii) We also consider the coupling function $\rho(x)$ without compact support. Respectively, for the proof of the time decay for the linearized equation, we use the Jensen-Kato results [14, 15] and the Agmon weighted norms [1].

Remark 1.1. The term $m^{2}$ in Schrödinger equation appears automatically in the nonrelativistic limit of the Klein-Gordon equation, and traditionally is removed by a gauge transformation. We keep the term to provide the existence of the nonzero solitons.

\section{Main Results}

\subsection{Existence of Dynamics}

To formulate our results precisely, we need some definitions. We introduce a suitable phase space for the Cauchy problem corresponding to (1.2) and (1.3). Let $H^{0}=L^{2}$, and $H^{1}$ be the Sobolev space $H^{1}=\left\{\psi \in L^{2}:|\nabla \psi| \in L^{2}\right\}$ with the norm $\|\psi\|_{H^{1}}=\|\nabla \psi\|_{L^{2}}+\|\psi\|_{L^{2}}$. Let us introduce also the weighted Sobolev spaces $H_{\alpha}^{s}, s=0,1, \alpha \in \mathbb{R}$ with the norms $\|\psi\|_{s, \alpha}:=\left\|(1+|x|)^{\alpha} \psi\right\|_{H^{s}}$.

Definition 2.1. i) The phase space $\mathcal{E}$ is the real Hilbert space $H^{1} \oplus H^{1} \oplus \mathbb{R}^{3} \oplus \mathbb{R}^{3}$ of states $Y=\left(\psi_{1}, \psi_{2}, q, p\right)$ with the finite norm

$$
\|Y\|_{\mathcal{E}}=\left\|\psi_{1}\right\|_{H^{1}}+\left\|\psi_{2}\right\|_{H^{1}}+|q|+|p| .
$$

ii) $\mathcal{E}_{\alpha}$ is the space $H_{\alpha}^{1} \oplus H_{\alpha}^{1} \oplus \mathbb{R}^{3} \oplus \mathbb{R}^{3}$ with the norm

$$
\|Y\|_{\alpha}=\|Y\|_{\mathcal{E}_{\alpha}}=\left\|\psi_{1}\right\|_{1, \alpha}+\left\|\psi_{2}\right\|_{1, \alpha}+|q|+|p| .
$$

iii) $\mathcal{E}^{+}$is space $H^{2} \oplus H^{2} \oplus \mathbb{R}^{3} \oplus \mathbb{R}^{3}$ with the norm

$$
\|Y\|_{\mathcal{E}}^{+}=\left\|\psi_{1}\right\|_{H^{2}}+\left\|\psi_{2}\right\|_{H^{2}}+|q|+|p| .
$$


For $\psi_{j} \in L^{2}$ we have

$$
-\frac{1}{2 m^{2}}\left\|\rho_{j}\right\|_{L^{2}}^{2} \leq \frac{m^{2}}{2}\left\|\psi_{j}\right\|_{L^{2}}^{2}+\left\langle\psi_{j}, \rho_{j}(\cdot-q)\right\rangle \leq \frac{m^{2}+1}{2}\left\|\psi_{j}\right\|_{L^{2}}^{2}+\frac{1}{2}\left\|\rho_{j}\right\|_{L^{2}}^{2} .
$$

Therefore $\mathcal{E}$ is the space of finite energy states. The Hamilton functional $\mathcal{H}$ is continuous on the space $\mathcal{E}$ and the lower bound in (2.1) implies that the energy (1.3) is bounded from below.

The system (1.2) reads as the Hamilton system

$$
\dot{Y}=J \mathcal{D H}(Y), \quad J:=\left(\begin{array}{cccc}
0 & 1 & 0 & 0 \\
-1 & 0 & 0 & 0 \\
0 & 0 & 0 & 1 \\
0 & 0 & -1 & 0
\end{array}\right), \quad Y=\left(\psi_{1}, \psi_{2}, q, p\right) \in \mathcal{E},
$$

where $\mathcal{D H}$ is the Fréchet derivative of the Hamilton functional (1.3).

Proposition 2.1. Let (1.9) hold. Then

(i) For every $Y_{0} \in \mathcal{E}$ the Cauchy problem (1.4) has a unique solution $Y(t) \in C(\mathbb{R}, \mathcal{E})$.

(ii) For every $t \in \mathbb{R}$, the map $U(t): Y_{0} \mapsto Y(t)$ is continuous on $\mathcal{E}$.

(iii) The energy is conserved, i.e.

$$
\mathcal{H}(Y(t))=\mathcal{H}\left(Y_{0}\right), \quad t \in \mathbb{R}
$$

Proof. Step i) Let us fix an arbitrary $b>0$ and prove (i)-(iii) for $Y_{0} \in \mathcal{E}$ such that $\left\|Y_{0}\right\|_{\mathcal{E}} \leq b$ and $|t| \leq \varepsilon=\varepsilon(b)$ for some sufficiently small $\varepsilon(b)>0$. Let us rewrite the Cauchy problem (1.4) us

$$
\dot{Y}(t)=F_{1}(Y(t))+F_{2}(Y(t)), \quad t \in \mathbb{R}: \quad Y(0)=Y_{0},
$$

where $F_{1}: Y \mapsto\left(\left(-\Delta+m^{2}\right) \Psi_{2},\left(\Delta-m^{2}\right) \Psi_{1}, 0,0\right)$. The Fourier transform provides the existence and uniqueness of solution $Y_{1}(t) \in C(\mathbb{R}, \mathcal{E})$ to the linear problem (2.4) with $F_{2}=0$. Let $U_{1}(t): Y_{0} \mapsto Y_{1}(t)$ be the corresponding strongly continuous group of bounded linear operators on $\mathcal{E}$. Then $(2.4)$ for $Y(t) \in C(\mathbb{R}, \mathcal{E})$ is equivalent to

$$
Y(t)=U_{1}(t) Y_{0}+\int_{0}^{t} d s U_{1}(t-s) F_{2}(Y(s))
$$

because $F_{2}(Y(\cdot)) \in C(\mathbb{R}, \mathcal{E})$ in this case. The latter follows from a local Lipschitz continuity of the map $F_{2}$ in $\mathcal{E}$ : for each $b>0$ there exist a $\varkappa=\varkappa(b)>0$ such that for all $Y, Z \in \mathcal{E}$ with $\|Y\|_{\mathcal{E}},\|Z\|_{\mathcal{E}} \leq b$

$$
\left\|F_{2}(Y)-F_{2}(Z)\right\|_{\mathcal{E}} \leq \varkappa\|Y-Z\|_{\mathcal{E}}
$$

Therefore, by the contraction mapping principle, equation (2.5) has a unique local solution $Y(\cdot) \in C([-\varepsilon, \varepsilon], \mathcal{E})$ with $\varepsilon>0$ depending only on $b$.

Step ii) We use now energy conservation to ensure the existence of a global solution and its continuity. First consider $Y_{0} \in \mathcal{E}_{c}:=C_{0}^{\infty} \oplus C_{0}^{\infty} \oplus \mathbb{R}^{3} \oplus \mathbb{R}^{3}$. Then $Y(t) \in \mathcal{E}^{+}$since $U_{1}(t) Y_{0}, F_{2}(Y(t)) \in \mathcal{E}^{+}$by (1.9). The energy conservation law follows by (2.2) and the the chain rule for the Fréchet derivatives:

$$
\frac{d}{d t} \mathcal{H}(Y(t))=\langle D \mathcal{H}(Y(t)), \dot{Y}(t)\rangle=\langle D \mathcal{H}(Y(t)), J D \mathcal{H}(Y(t))\rangle=0, \quad t \in \mathbb{R}
$$


since the operator $J$ is skew-symmetric by $(2.2)$, and $D \mathcal{H}(Y(t)) \in L^{2} \oplus L^{2} \oplus \mathbb{R}^{3} \oplus \mathbb{R}^{3}$ for $Y(t) \in \mathcal{E}^{+}$. The inequality (2.1) implies

$$
\mathcal{H} \geq \frac{1}{2}\|\nabla \psi\|_{L^{2}}^{2}+\frac{m^{2}}{4}\|\psi\|_{L^{2}}^{2}+\frac{1}{2}|p|^{2}-\frac{1}{m^{2}}\|\rho\|_{L^{2}}^{2}
$$

Hence, by energy conservation, for $|t| \leq \varepsilon$

$$
\frac{1}{2}\|\nabla \psi\|_{L^{2}}^{2}+\frac{m^{2}}{4}\|\psi\|_{L^{2}}^{2}+\frac{1}{2}|p|^{2}-\frac{1}{m^{2}}\|\rho\|_{L^{2}}^{2} \leq \mathcal{H}(Y(t))=\mathcal{H}\left(Y_{0}\right)
$$

This implies a priori estimate

$$
\|\psi\|_{H^{1}}+|p| \leq B \quad \text { for }|t| \leq \varepsilon
$$

with $B$ depending only on the norm $\left\|Y_{0}\right\|_{\mathcal{E}}$ of the initial data and on $\|\rho\|_{L^{2}}$. An arbitrary initial data $Y_{0} \in \mathcal{E}$ can be approximated by initial data from $\mathcal{E}_{c}$. The corresponding solution exists due to representation (2.5) by contraction mapping principle, and then (2.6) follows by the limit transition.

Step iii) Properties (i)-(iii) for arbitrary $t \in \mathbb{R}$ now follow from the same properties for small $|t|$ and from a priori bound (2.6).

\subsection{Solitary Manifold and Main Result}

Let us compute the solitons (1.5). The substitution to (1.1) gives the following stationary equations

$$
\begin{aligned}
& -i v \cdot \nabla \psi_{v}(y)=\left(-\Delta+m^{2}\right) \psi_{v}(y)+\rho(y) \\
& p=v, \quad 0=-\int\left(\overline{\nabla \psi}_{v}(y) \rho(y)+\nabla \psi_{v}(y) \bar{\rho}(y)\right) d y
\end{aligned}
$$

Then the first equation implies

$$
\Lambda \psi_{v}(y):=\left[-\Delta+m^{2}+i v \cdot \nabla\right] \psi_{v}(y)=-\rho(y), \quad y \in \mathbb{R}^{3} .
$$

For $|v|<2 m$ the operator $\Lambda$ is an isomorphism $H^{4}\left(\mathbb{R}^{3}\right) \rightarrow H^{2}\left(\mathbb{R}^{3}\right)$. Hence (1.9) implies that

$$
\psi_{v}(y)=-\Lambda^{-1} \rho(y) \in H^{4}\left(\mathbb{R}^{3}\right) .
$$

If $v$ is given and $|v|<2 m$, then $p_{v}$ can be found from the second equation of (2.7).

The function $\psi_{v}$ can be computed by the Fourier transform. The soliton is given by the formula

$$
\psi_{v}(x)=-\frac{1}{4 \pi} \int \frac{e^{-\sqrt{m^{2}-\frac{v^{2}}{4}}|x-y|} e^{i \frac{v}{2}(x-y)} \rho(y) d^{3} y}{|x-y|} .
$$

Further, in Appendix A, we prove that the last equation of of (2.7) holds. Hence, the soliton solution (1.5) exists and defined uniquely for any couple $(a, v)$ with $|v|<2 \mathrm{~m}$. Let us denote by $V:=\left\{v \in \mathbb{R}^{3}:|v|<2 m\right\}, \psi_{v 1}=\operatorname{Re} \psi_{v}$, and $\psi_{v 2}=\operatorname{Im} \psi_{v}$.

Definition 2.2. A soliton state is $S(\sigma):=\left(\psi_{v 1}(x-b), \psi_{v 2}(x-b), b, v\right)$, where $\sigma:=(b, v)$ with $b \in \mathbb{R}^{3}$ and $v \in V$.

Obviously, the soliton solution admits the representation $S(\sigma(t))$, where

$$
\sigma(t)=(b(t), v(t))=(v t+a, v) .
$$

Definition 2.3. A solitary manifold is the set $\mathcal{S}:=\left\{S(\sigma): \sigma \in \Sigma:=\mathbb{R}^{3} \times V\right\}$. 
The main result of our paper is the following theorem.

Theorem 2.1. Let (1.9), and the Wiener condition (1.10) hold. Let $\beta>3 / 2$ be the number from (1.9), and $Y(t)$ be the solution to the Cauchy problem (1.4) with the initial state $Y_{0}$ which is sufficiently close to the solitary manifold:

$$
p_{0}<2 m, \quad d_{0}:=\operatorname{dist}_{\mathcal{E}_{\beta}}\left(Y_{0}, \mathcal{S}\right) \ll 1 .
$$

Then the asymptotics hold for $t \rightarrow \pm \infty$,

$$
\begin{gathered}
\dot{q}(t)=v_{ \pm}+\mathcal{O}\left(|t|^{-2}\right), \quad q(t)=v_{ \pm} t+a_{ \pm}+\mathcal{O}\left(|t|^{-3 / 2}\right) \\
\psi(x, t)=\psi_{v \pm}\left(x-v_{ \pm} t-a_{ \pm}\right)+W_{0}(t) \boldsymbol{\psi}_{ \pm}+r_{ \pm}(x, t)
\end{gathered}
$$

with

$$
\left\|r_{ \pm}(t)\right\|_{H^{1}}=\mathcal{O}\left(|t|^{-1 / 2}\right)
$$

It suffices to prove the asymptotics (2.13), (2.14) for $t \rightarrow+\infty$ since the system (1.2) is time reversible.

\section{$3 \quad$ Symplectic Projection onto Solitary Manifold}

Let us identify the tangent space to $\mathcal{E}$, at every point, with $\mathcal{E}$. Consider the symplectic form $\Omega$ defined on $\mathcal{E}$ by $\Omega=\int d \psi_{1}(x) \wedge d \psi_{2}(x) d x+d q \wedge d p$, i.e.

$$
\Omega\left(Y_{1}, Y_{2}\right)=\left\langle Y_{1}, J Y_{2}\right\rangle, \quad Y_{1}, Y_{2} \in \mathcal{E}
$$

where

$$
\left\langle Y_{1}, Y_{2}\right\rangle:=\left\langle\psi_{11}, \psi_{12}\right\rangle+\left\langle\psi_{21}, \psi_{22}\right\rangle+q_{1} q_{2}+p_{1} p_{2}
$$

and $\left\langle\psi_{11}, \psi_{12}\right\rangle=\int \psi_{11}(x) \psi_{12}(x) d x$ etc. It is clear that the form $\Omega$ is non-degenerate, i.e.

$$
\Omega\left(Y_{1}, Y_{2}\right)=0 \text { for every } Y_{2} \in \mathcal{E} \Longrightarrow Y_{1}=0
$$

Definition 3.1. i) $Y_{1} \nmid Y_{2}$ means that $Y_{1} \in \mathcal{E}, Y_{2} \in \mathcal{E}$, and $Y_{1}$ is symplectic orthogonal to $Y_{2}$, i.e. $\Omega\left(Y_{1}, Y_{2}\right)=0$.

ii) A projection operator $\mathbf{P}: \mathcal{E} \rightarrow \mathcal{E}$ is called symplectic orthogonal if $Y_{1} \nmid Y_{2}$ for $Y_{1} \in \operatorname{Ker} \mathbf{P}$ and $Y_{2} \in \operatorname{Im} \mathbf{P}$.

Let us consider the tangent space $\mathcal{T}_{S(\sigma)} \mathcal{S}$ to the manifold $\mathcal{S}$ at a point $S(\sigma)$. The vectors $\tau_{j}:=\partial_{\sigma_{j}} S(\sigma)$, where $\partial_{\sigma_{j}}:=\partial_{b_{j}}$ and $\partial_{\sigma_{j+3}}:=\partial_{v_{j}}$ with $j=1,2,3$, form a basis in $\mathcal{T}_{\sigma} \mathcal{S}$. In detail,

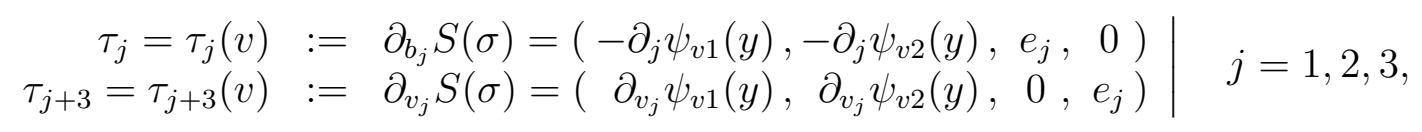

where $y:=x-b$ is the "moving frame coordinate", $e_{1}=(1,0,0)$ etc. Let us stress that the functions $\tau_{j}$ will be considered always as the functions of $y$, not of $x$.

The formulas (2.10) and the conditions (1.9) imply that

$$
\tau_{j}(v) \in \mathcal{E}_{\alpha}, \quad v \in V, \quad j=1, \ldots, 6, \quad \forall \alpha \leq \beta
$$


Lemma 3.1. The matrix with the elements $\Omega\left(\tau_{l}(v), \tau_{j}(v)\right)$ is non-degenerate for any $v \in$ $V$.

Proof The elements are computed in Appendix B. As the result, the matrix $\Omega\left(\tau_{l}, \tau_{j}\right)$ has the form

$$
\Omega(v):=\left(\Omega\left(\tau_{l}, \tau_{j}\right)\right)_{l, j=1, \ldots, 6}=\left(\begin{array}{ll}
0 & \Omega^{+}(v) \\
-\Omega^{+}(v) & 0
\end{array}\right),
$$

where the $3 \times 3$-matrix $\Omega^{+}(v)$ equals

$$
\Omega^{+}(v)=K+E
$$

Here $K$ is a symmetric $3 \times 3$-matrix with the elements

$$
K_{i j}=\int \frac{k_{j} k_{l}\left(\left(k^{2}+m^{2}\right)\left(\left|\hat{\psi}_{v 1}\right|^{2}+\left|\hat{\psi}_{v 2}\right|^{2}\right)+i(k v)\left(\hat{\psi}_{v 1} \overline{\hat{\psi}}_{v 2}-\hat{\psi}_{v 2} \overline{\hat{\psi}}_{v 1}\right)\right) d k}{\left(k^{2}+m^{2}\right)^{2}-(k v)^{2}}
$$

where the "hat" denotes the Fourier transform (cf. (1.10)). The matrix $K$ is the integral of the symmetric nonnegative definite matrix $k \otimes k=\left(k_{i} k_{j}\right)$ with a nonnegative weight. (The last statement is true since $k^{2}+m^{2}>|(k v)|$ for $|v|<2 m$, and $\left|\hat{\psi}_{v 1}+i \hat{\psi}_{v 2}\right|^{2}=$ $\left|\hat{\psi}_{v 1}\right|^{2}+\left|\hat{\psi}_{v 2}\right|^{2}-i\left(\hat{\psi}_{v 1} \overline{\hat{\psi}}_{v 2}-\hat{\psi}_{v 2} \overline{\hat{\psi}}_{v 1}\right) \geq 0$.) Hence, the matrix $K$ is also nonnegative definite. Since the unite matrix $E$ is positive definite, the matrix $\Omega^{+}(v)$ is symmetric and positive definite, hence non-degenerate. Then the matrix $\Omega\left(\tau_{l}, \tau_{j}\right)$ also is non-degenerate.

Let us introduce the translations $T_{a}:\left(\psi_{1}(\cdot), \psi_{2}(\cdot), q, p\right) \mapsto\left(\psi_{1}(\cdot-a), \psi_{2}(\cdot-a), q+a, p\right)$, $a \in \mathbb{R}^{3}$. Note that the manifold $\mathcal{S}$ is invariant with respect to the translations.

Definition 3.2. i) For any $\alpha \in \mathbb{R}$ and $\bar{p}<2 m$ denote by $\mathcal{E}_{\alpha}(\bar{p})=\left\{Y=\left(\psi_{1}, \psi_{2}, q, p\right) \in\right.$ $\left.\mathcal{E}_{\alpha}:|p| \leq \bar{p}\right\}$. We set $\mathcal{E}(\bar{p}):=\mathcal{E}_{0}(\bar{p})$.

ii) For any $\bar{v}<2 m$ denote by $\Sigma(\bar{v})=\left\{\sigma=(b, v): b \in \mathbb{R}^{3},|v| \leq \bar{v}\right\}$.

The next Lemma provide that in a small neighborhood of the soliton manifold $\mathcal{S}$ a "symplectic orthogonal projection" onto $\mathcal{S}$ is well-defined. The proof is similar to the proof of the Lemma 3.4 in [10].

Lemma 3.2. Let (1.9) hold, $\alpha \in \mathbb{R}$. Then

i) there exists a neighborhood $\mathcal{O}_{\alpha}(\mathcal{S})$ of $\mathcal{S}$ in $\mathcal{E}_{\alpha}$ and a map $\Pi: \mathcal{O}_{\alpha}(\mathcal{S}) \rightarrow \mathcal{S}$ such that $\Pi$ is uniformly continuous in the metric of $\mathcal{E}_{\alpha}$ on $\mathcal{O}_{\alpha}(\mathcal{S}) \cap \mathcal{E}_{\alpha}(\bar{p})$ with $\bar{p}<2 m$,

$$
\Pi Y=Y \quad \text { for } Y \in \mathcal{S}, \quad \text { and } \quad Y-S \nmid \mathcal{T}_{S} \mathcal{S}, \text { where } S=\Pi Y .
$$

ii) $\mathcal{O}_{\alpha}(\mathcal{S})$ is invariant with respect to the translations $T_{a}$, and

$$
\Pi T_{a} Y=T_{a} \Pi Y, \quad \text { for } Y \in \mathcal{O}_{\alpha}(\mathcal{S}) \text { and } a \in \mathbb{R}^{3} \text {. }
$$

iii) For any $\bar{p}<2 m$ there exists a $\bar{v}<2 m$ s.t. $\Pi Y=S(\sigma)$ with $\sigma \in \Sigma(\bar{v})$ for $Y \in$ $\mathcal{O}_{\alpha}(\mathcal{S}) \cap \mathcal{E}_{\alpha}(\bar{p})$

iv) For any $\bar{v}<2 m$ there exists an $r_{\alpha}(\bar{v})>0$ s.t. $S(\sigma)+Z \in \mathcal{O}_{\alpha}(\mathcal{S})$ if $\sigma \in \Sigma(\bar{v})$ and $\|Z\|_{\alpha}<r_{\alpha}(\bar{v})$.

We will call $\boldsymbol{\Pi}$ a symplectic orthogonal projection onto $\mathcal{S}$.

Corollary 3.1. The condition (2.12) implies that $Y_{0}=S+Z_{0}$ where $S=S\left(\sigma_{0}\right)=\Pi Y_{0}$, and

$$
\left\|Z_{0}\right\|_{\beta} \ll 1 .
$$


Proof Lemma 3.2 implies that $\Pi Y_{0}=S$ is well defined for small $d_{0}>0$. Furthermore, the condition (2.12) means that there exists a point $S_{1} \in \mathcal{S}$ such that $\left\|Y_{0}-S_{1}\right\|_{\beta}=d_{0}$. Hence, $Y_{0}, S_{1} \in \mathcal{O}_{\beta}(\mathcal{S}) \cap \mathcal{E}_{\beta}(\bar{p})$ with a $\bar{p}<2 m$ which does not depend on $d_{0}$ for sufficiently small $d_{0}$. On the other hand, $\Pi S_{1}=S_{1}$, hence the uniform continuity of the map $\Pi$ implies that $\left\|S_{1}-S\right\|_{\beta} \rightarrow 0$ as $d_{0} \rightarrow 0$. Therefore, finally, $\left\|Z_{0}\right\|_{\beta}=\left\|Y_{0}-S\right\|_{\beta} \leq$ $\left\|Y_{0}-S_{1}\right\|_{\beta}+\left\|S_{1}-S\right\|_{\beta} \leq d_{0}+o(1) \ll 1$ for small $d_{0}$.

\section{Linearization on the Solitary Manifold}

Let us consider a solution to the system (1.2), and split it as the sum

$$
Y(t)=S(\sigma(t))+Z(t)
$$

where $\sigma(t)=(b(t), v(t)) \in \Sigma$ is an arbitrary smooth function of $t \in \mathbb{R}$. In detail, denote $Y=\left(\psi_{1}, \psi_{2}, q, p\right)$ and $Z=\left(\Psi_{1}, \Psi_{2}, Q, P\right)$. Then (4.1) means that

$$
\begin{aligned}
& \psi_{1}(x, t)=\psi_{v(t) 1}(x-b(t))+\Psi_{1}(x-b(t), t), \quad q(t)=b(t)+Q(t) \\
& \psi_{2}(x, t)=\psi_{v(t) 2}(x-b(t))+\Psi_{2}(x-b(t), t), \quad p(t)=v(t)+P(t)
\end{aligned}
$$

Let us substitute (4.2) to (1.2), and linearize the equations in $Z$. Later we will choose $S(\sigma(t))=\Pi Y(t)$, i.e. $Z(t)$ is symplectic orthogonal to $\mathcal{T}_{S(\sigma(t))} \mathcal{S}$.

Setting $y=x-b(t)$ which is the "moving frame coordinate", we obtain from $(4.2)$ and (1.2) that

$$
\begin{aligned}
\dot{\psi}_{1} & =\dot{v} \cdot \nabla_{v} \psi_{v 1}(y)-\dot{b} \cdot \nabla \psi_{v 1}(y)+\dot{\Psi}_{1}(y, t)-\dot{b} \cdot \nabla \Psi_{1}(y, t) \\
& =-\Delta \psi_{v 2}(y)+m^{2} \psi_{v 2}(y)-\Delta \Psi_{2}(y, t)+m^{2} \Psi_{2}(y, t)+\rho_{2}(y-Q) \\
\dot{\psi}_{2} & =\dot{v} \cdot \nabla_{v} \psi_{v 2}(y)-\dot{b} \cdot \nabla \psi_{v 2}(y)+\dot{\Psi}_{2}(y, t)-\dot{b} \cdot \nabla \Psi_{2}(y, t) \\
& =\Delta \psi_{v 1}(y)-m^{2} \psi_{v 1}(y)+\Delta \Psi_{1}(y, t)-m^{2} \Psi_{1}(y, t)-\rho_{1}(y-Q) \\
\dot{q} & =\dot{b}+\dot{Q}=v+P \\
\dot{p} & =\dot{v}+\dot{P}=-\left\langle\nabla\left(\psi_{v j}(y)+\Psi_{j}(y, t)\right), \rho_{j}(y-Q)\right\rangle
\end{aligned}
$$

Let us to extract linear terms in $Q$. First note that $\rho_{j}(y-Q)=\rho_{j}(y)-Q \cdot \nabla \rho_{j}(y)+$ $N_{j}(Q), j=1,2$, where $N_{j}(Q)=\rho_{j}(y-Q)-\rho_{j}(y)+Q \cdot \nabla \rho_{j}(y)$. The condition (1.9) implies that for $N_{j}(Q)$ the bound holds,

$$
\left\|N_{j}(Q)\right\|_{0, \beta} \leq C_{\beta}(\bar{Q}) Q^{2}, j=1,2
$$

uniformly in $|Q| \leq \bar{Q}$ for any fixed $\bar{Q}$, where $\beta$ is the parameter from Theorem 2.1. Using the equations (2.7), we obtain from (4.3) the following equations for the components of 
the vector $Z(t)$ :

$$
\begin{aligned}
\dot{\Psi}_{1}(y, t) & =-\Delta \Psi_{2}(y, t)+m^{2} \Psi_{2}(y, t)+\dot{b} \cdot \nabla \Psi_{1}(y, t)-Q \cdot \nabla \rho_{2}(y) \\
& +(\dot{b}-v) \cdot \nabla \psi_{v 1}(y)-\dot{v} \cdot \nabla_{v} \psi_{v 1}(y)+N_{2} \\
\dot{\Psi}_{2}(y, t) & =\Delta \Psi_{1}(y, t)-m^{2} \Psi_{1}(y, t)+\dot{b} \cdot \nabla \Psi_{2}(y, t)+Q \cdot \nabla \rho_{1}(y) \\
& +(\dot{b}-v) \cdot \nabla \psi_{v 2}(y)-\dot{v} \cdot \nabla_{v} \psi_{v 2}(y)-N_{1} \\
\dot{Q}(t) & =P+(v-\dot{b}) \\
\dot{P}(t) & =\left\langle\Psi_{j}(y, t), \nabla \rho_{j}(y)\right\rangle+\left\langle\nabla \psi_{v j}(y), Q \cdot \nabla \rho_{j}(y)\right\rangle-\dot{v}+N_{4}(v, Z)
\end{aligned}
$$

where $N_{4}(v, Z)=-\left\langle\nabla \psi_{v j}, N_{j}(Q)\right\rangle+\left\langle\nabla \Psi_{j}, Q \cdot \nabla \rho_{j}\right\rangle-\left\langle\nabla \Psi_{j}, N_{j}(Q)\right\rangle$. Clearly, $N_{4}(v, Z)$ satisfies the following estimate

$$
\left|N_{4}(v, Z)\right| \leq C_{\beta}(\rho, \bar{v}, \bar{Q})\left[Q^{2}+\left\|\Psi_{1}\right\|_{1,-\beta}|Q|+\left\|\Psi_{2}\right\|_{1,-\beta}|Q|\right],
$$

uniformly in $|v| \leq \bar{v}$ and $|Q| \leq \bar{Q}$ for any fixed $\bar{v}<2 m$. We can write the equations (4.5) as

$$
\dot{Z}(t)=A(t) Z(t)+T(t)+N(t), \quad t \in \mathbb{R} .
$$

Here the operator $A(t)=A_{v, w}(t)$ depends on two parameters, $v=v(t)$, and $w:=\dot{b}(t)$ and can be written in the form

$$
A_{v, w}\left(\begin{array}{l}
\Psi_{1} \\
\Psi_{2} \\
Q \\
P
\end{array}\right)=\left(\begin{array}{cccc}
w \cdot \nabla & -\left(\Delta-m^{2}\right) & -\nabla \rho_{2} \cdot & 0 \\
\Delta-m^{2} & w \cdot \nabla & \nabla \rho_{1} \cdot & 0 \\
0 & 0 & 0 & E \\
\left\langle\cdot, \nabla \rho_{1}\right\rangle & \left\langle\cdot, \nabla \rho_{2}\right\rangle & \left\langle\nabla \psi_{v j}, \cdot \nabla \rho_{j}\right\rangle & 0
\end{array}\right)\left(\begin{array}{l}
\Psi_{1} \\
\Psi_{2} \\
Q \\
P
\end{array}\right) .
$$

Furthermore, $T(t)=T_{v, w}(t)$ and $N(t)=N(t, \sigma, Z)$ in (4.7) stand for

$$
T_{v, w}=\left(\begin{array}{c}
(w-v) \cdot \nabla \psi_{v 1}-\dot{v} \cdot \nabla_{v} \psi_{v 1} \\
(w-v) \cdot \nabla \psi_{v 2}-\dot{v} \cdot \nabla_{v} \psi_{v 2} \\
v-w \\
-\dot{v}
\end{array}\right), \quad N(\sigma, Z)=\left(\begin{array}{c}
N_{2}(Z) \\
-N_{1}(Z) \\
0 \\
N_{4}(v, Z)
\end{array}\right)
$$

where $v=v(t), w=w(t), \sigma=\sigma(t)=(b(t), v(t))$, and $Z=Z(t)$. The estimates (4.4) and (4.6) imply that

$$
\|N(\sigma, Z)\|_{\beta} \leq C(\bar{v}, \bar{Q})\|Z\|_{-\beta}^{2},
$$

uniformly in $\sigma \in \Sigma(\bar{v})$ and $\|Z\|_{-\beta} \leq r_{-\beta}(\bar{v})$ for any fixed $\bar{v}<2 m$.

Remark 4.1. i) The term $A(t) Z(t)$ in the right hand side of the equation (4.7) is linear in $Z(t)$, and $N(t)$ is a high order term in $Z(t)$. On the other hand, $T(t)$ is a zero order term which does not vanish at $Z(t)=0$ since $S(\sigma(t))$ generally is not a soliton solution if (2.11) does not hold (though $S(\sigma(t))$ belongs to the solitary manifold).

ii) Formulas (3.2) and (4.9) imply:

$$
T(t)=-\sum_{l=1}^{3}\left[(w-v){ }_{l} \tau_{l}+\dot{v}_{l} \tau_{l+3}\right]
$$

and hence $T(t) \in \mathcal{T}_{S(\sigma(t))} \mathcal{S}, t \in \mathbb{R}$. This fact suggests an unstable character of the nonlinear dynamics along the solitary manifold. 
Here we collect some Hamiltonian and spectral properties of the generator (4.8) of the linearized equation. First, let us consider the linear equation

$$
\dot{X}(t)=A_{v, w} X(t), \quad t \in \mathbb{R}, \quad v \in V, \quad w \in \mathbb{R}^{3}
$$

Lemma 5.1. (cf. [10]) i) For any $v \in V$ and $w \in \mathbb{R}^{3}$ the equation (5.1) can be written as the Hamilton system (cf. (2.2)),

$$
\dot{X}(t)=J D \mathcal{H}_{v, w}(X(t)), \quad t \in \mathbb{R}
$$

where $D \mathcal{H}_{v, w}$ is the Fréchet derivative of the Hamilton functional

$$
\begin{aligned}
& \mathcal{H}_{v, w}(X)=\frac{1}{2} \int\left[\left|\nabla \Psi_{1}\right|^{2}+m^{2}\left|\Psi_{1}\right|^{2}+\left|\nabla \Psi_{2}\right|^{2}+m^{2}\left|\Psi_{2}\right|^{2}\right] d y+\int \Psi_{2} w \cdot \nabla \Psi_{1} d y \\
& +\int \rho_{j}(y) Q \cdot \nabla \Psi_{j} d y+\frac{1}{2} P^{2}-\frac{1}{2}\left\langle Q \cdot \nabla \psi_{v j}(y), Q \cdot \nabla \rho_{j}(y)\right\rangle, \quad X=\left(\Psi_{1}, \Psi_{2}, Q, P\right) \in \mathcal{E},
\end{aligned}
$$

ii) Energy conservation law holds for the solutions $X(t) \in C^{1}\left(\mathbb{R}, \mathcal{E}^{+}\right)$,

$$
\mathcal{H}_{v, w}(X(t))=\text { const }, \quad t \in \mathbb{R}
$$

iii) The skew-symmetry relation holds,

$$
\Omega\left(A_{v, w} X_{1}, X_{2}\right)=-\Omega\left(X_{1}, A_{v, w} X_{2}\right), \quad X_{1}, X_{2} \in \mathcal{E}
$$

iv) The operator $A_{v, w}$ acts on the tangent vectors $\tau_{j}(v)$ to the solitary manifold as follows,

$$
A_{v, w}\left[\tau_{j}(v)\right]=(w-v) \cdot \nabla \tau_{j}(v), \quad A_{v, w}\left[\tau_{j+3}(v)\right]=(w-v) \cdot \nabla \tau_{j+3}(v)+\tau_{j}(v), j=1,2,3
$$

We will apply Lemma 5.1 mainly to the operator $A_{v, v}$ corresponding to $w=v$. In that case the linearized equation has the following additional essential features.

Lemma 5.2. Let us assume that $w=v \in V$. Then

i) The tangent vectors $\tau_{j}(v)$ with $j=1,2,3$ are eigenvectors, and $\tau_{j+3}(v)$ are root vectors of the operator $A_{v, v}$, corresponding to zero eigenvalue, i.e.

$$
A_{v, v}\left[\tau_{j}(v)\right]=0, \quad A_{v, v}\left[\tau_{j+3}(v)\right]=\tau_{j}(v), \quad j=1,2,3 .
$$

ii) The Hamilton function (5.3) is nonnegative definite since

$$
\mathcal{H}_{v, v}(X)=\frac{1}{2} \int\left|\Lambda^{1 / 2}\left(\Psi_{1}+i \Psi_{2}\right)-\Lambda^{-1 / 2} Q \cdot \nabla\left(\rho_{1}+i \rho_{2}\right)\right|^{2} d x+\frac{1}{2} P^{2} \geq 0 .
$$

Here $\Lambda$ is the operator (2.8) which is symmetric and nonnegative definite in $L^{2}\left(\mathbb{R}^{3}\right)$ for $|v|<2 m$, and $\Lambda^{1 / 2}$ is the nonnegative definite square root defined in the Fourier representation. 
Proof. The first statement follows from (5.6) with $w=v$. In order to prove ii) we rewrite the integral in (5.8) as follows:

$$
\begin{aligned}
& \frac{1}{2}\left\langle\Lambda^{1 / 2}\left(\Psi_{1}+i \Psi_{2}\right)-\Lambda^{-1 / 2} Q \cdot \nabla\left(\rho_{1}+i \rho_{2}\right), \Lambda^{1 / 2}\left(\Psi_{1}+i \Psi_{2}\right)-\Lambda^{-1 / 2} Q \cdot \nabla\left(\rho_{1}+i \rho_{2}\right)\right\rangle \\
& =\frac{1}{2}\left\langle\Lambda\left(\Psi_{1}+i \Psi_{2}\right), \Psi_{1}+i \Psi_{2}\right\rangle-\left\langle\Psi_{j}, Q \cdot \nabla \rho_{j}\right\rangle+\frac{1}{2}\left\langle\Lambda^{-1} Q \cdot \nabla\left(\rho_{1}+i \rho_{2}\right), Q \cdot \nabla\left(\rho_{1}+i \rho_{2}\right)\right\rangle
\end{aligned}
$$

since the operator $\Lambda^{1 / 2}$ is symmetric in $L^{2}\left(\mathbb{R}^{3}\right)$. Now all the terms of the expression (5.9) can be identified with the corresponding terms in (5.3) since

$$
\begin{aligned}
\frac{1}{2}\left\langle\Lambda\left(\Psi_{1}+i \Psi_{2}\right), \Psi_{1}+i \Psi_{2}\right\rangle & =\frac{1}{2}\left\langle\left[-\Delta+m^{2}+i v \cdot \nabla\right]\left(\Psi_{1}+i \Psi_{2}\right),\left(\Psi_{1}+i \Psi_{2}\right)\right\rangle \\
= & \frac{1}{2}\left\langle\left[-\Delta+m^{2}\right] \Psi_{1}, \Psi_{1}\right\rangle+\frac{1}{2}\left\langle\left[-\Delta+m^{2}\right] \Psi_{2}, \Psi_{2}\right\rangle+\left\langle\Psi_{2}, v \cdot \nabla \Psi_{1}\right\rangle
\end{aligned}
$$

and $\Lambda^{-1}\left(\rho_{1}+i \rho_{2}\right)=-\left(\psi_{v 1}+i \psi_{v 2}\right)$ by $(2.8)$ and $(2.9)$.

Remark 5.1. For a soliton solution of the system(1.2) we have $\dot{b}=v, \dot{v}=0$, and hence $T(t) \equiv 0$. Thus, the equation(5.1) is the linearization of the system (1.2) on a soliton solution. In fact, we do not linearize (1.2) on a soliton solution, but on a trajectory $S(\sigma(t))$ with $\sigma(t)$ being nonlinear in $t$. We will show later that $T(t)$ is quadratic in $Z(t)$ if we choose $S(\sigma(t))$ to be the symplectic orthogonal projection of $Y(t)$. Then (5.1) is again the linearization of (1.2).

\section{Symplectic Decomposition of the Dynamics}

Here we decompose the dynamics in two components: along the manifold $\mathcal{S}$ and in transversal directions. The equation (4.7) is obtained without any assumption on $\sigma(t)$ in (4.1). We are going to choose $S(\sigma(t)):=\Pi Y(t)$, but then we need to know that

$$
Y(t) \in \mathcal{O}_{-\beta}(\mathcal{S}), \quad t \in \mathbb{R}
$$

It is true for $t=0$ by our main assumption (2.12) with sufficiently small $d_{0}>0$. Then $S(\sigma(0))=\Pi Y(0)$ and $Z(0)=Y(0)-S(\sigma(0))$ are well defined. We will prove below that (6.1) holds if $d_{0}$ is sufficiently small. Let us choose an arbitrary $\bar{v}$ such that $|v(0)|<\bar{v}<2 m$ and let $\delta=\bar{v}-|v(0)|$. Denote by $r_{-\beta}(\bar{v})$ the positive numbers from Lemma 3.2 iv) which corresponds to $\alpha=-\beta$. Then $S(\sigma)+Z \in \mathcal{O}_{-\beta}(\mathcal{S})$ if $\sigma=(b, v)$ with $|v|<\bar{v}$ and $\|Z\|_{-\beta}<r_{-\beta}(\bar{v})$. Note that $\|Z(0)\|_{-\beta}<r_{-\beta}(\bar{v})$ if $d_{0}$ is sufficiently small. Therefore, $S(\sigma(t))=\Pi Y(t)$ and $Z(t)=Y(t)-S(\sigma(t))$ are well defined for $t \geq 0$ so small that $|v|<\bar{v}$ and $\|Z(t)\|_{-\beta}<r_{-\beta}(\bar{v})$. This is formalized by the following standard definition.

Definition 6.1. $t_{*}$ is the "exit time",

$$
t_{*}=\sup \left\{t>0:\|Z(s)\|_{-\beta}<r_{-\beta}(\bar{v}), \quad|v(s)-v(0)|<\delta, \quad 0 \leq s \leq t\right\} .
$$

One of our main goals is to prove that $t_{*}=\infty$ if $d_{0}$ is sufficiently small. This would follow if we show that

$$
\|Z(t)\|_{-\beta}<r_{-\beta}(\bar{v}) / 2, \quad|v(s)-v(0)|<\delta / 2, \quad 0 \leq t<t_{*} .
$$

Note that

$$
|Q(t)| \leq \bar{Q}:=r_{-\beta}(\bar{v}), \quad 0 \leq t<t_{*} .
$$

Now $N(t)$ in (4.7) satisfies, by (4.10), the following estimate,

$$
\|N(t)\|_{\beta} \leq C_{\beta}(\bar{v})\|Z(t)\|_{-\beta}^{2}, \quad 0 \leq t<t_{*} .
$$




\subsection{Longitudinal Dynamics: Modulation Equations}

From now on we fix the decomposition $Y(t)=S(\sigma(t))+Z(t)$ for $0<t<t_{*}$ by setting $S(\sigma(t))=\Pi Y(t)$ which is equivalent to the symplectic orthogonality condition of type $(3.7)$,

$$
Z(t) \nmid \mathcal{T}_{S(\sigma(t))} \mathcal{S}, \quad 0 \leq t<t_{*} .
$$

This allows us to simplify drastically the asymptotic analysis of the dynamical equations (4.7) for the transversal component $Z(t)$. As the first step, we derive the longitudinal dynamics, i.e. the "modulation equations" for the parameters $\sigma(t)$. Let us derive a system of ordinary differential equations for the vector $\sigma(t)$. For this purpose, let us write (6.6) in the form

$$
\Omega\left(Z(t), \tau_{j}(t)\right)=0, j=1, \ldots, 6, \quad 0 \leq t<t_{*},
$$

where the vectors $\tau_{j}(t)=\tau_{j}(\sigma(t))$ span the tangent space $\mathcal{T}_{S(\sigma(t))} \mathcal{S}$. Note that $\sigma(t)=$ $(b(t), v(t))$, where

$$
|v(t)| \leq \bar{v}<2 m, \quad 0 \leq t<t_{*},
$$

by Lemma $3.2 \mathrm{iii})$. It would be convenient for us to use some other parameters $(c, v)$ instead of $\sigma=(b, v)$, where $c(t)=b(t)-\int_{0}^{t} v(\tau) d \tau$ and

$$
\dot{c}(t)=\dot{b}(t)-v(t)=w(t)-v(t), \quad 0 \leq t<t_{*} .
$$

We do not need an explicit form of the equations for $(c, v)$ but the following statement, which can be proved similar to the Lemma 6.2 in [10].

Lemma 6.1. Let $Y(t)$ be a solution to the Cauchy problem (1.4), and (4.1), (6.7) hold. Then $(c(t), v(t))$ satisfies the equation

$$
\left(\begin{array}{c}
\dot{c}(t) \\
\dot{v}(t)
\end{array}\right)=\mathcal{N}(\sigma(t), Z(t)), \quad 0 \leq t<t_{*}
$$

where

$$
\mathcal{N}(\sigma, Z)=\mathcal{O}\left(\|Z\|_{-\beta}^{2}\right)
$$

uniformly in $\sigma \in \Sigma(\bar{v})$.

\subsection{Decay for the Transversal Dynamics}

In Section 11 we will show that our main Theorem 2.1 can be derived from the following time decay of the transversal component $Z(t)$ :

Proposition 6.1. Let all conditions of Theorem 2.1 hold. Then $t_{*}=\infty$, and

$$
\|Z(t)\|_{-\beta} \leq \frac{C\left(\rho, \bar{v}, d_{0}\right)}{(1+|t|)^{3 / 2}}, \quad t \geq 0 .
$$

We will derive (6.12) in Sections 7-11 from our equation (4.7) for the transversal component $Z(t)$. This equation can be specified using Lemma 6.1. Indeed, the lemma implies that

$$
\|T(t)\|_{\beta} \leq C(\bar{v})\|Z(t)\|_{-\beta}^{2}, \quad 0 \leq t<t_{*},
$$

by (4.9) since $w-v=\dot{c}$. Thus (4.7) becomes the equation

$$
\dot{Z}(t)=A(t) Z(t)+\tilde{N}(t), \quad 0 \leq t<t_{*},
$$


where $A(t)=A_{v(t), w(t)}$, and $\tilde{N}(t):=T(t)+N(t)$ satisfies the estimate

$$
\|\tilde{N}(t)\|_{\beta} \leq C\|Z(t)\|_{-\beta}^{2}, \quad 0 \leq t<t_{*} .
$$

In all remaining part of our paper we will analyze mainly the basic equation (6.14) to establish the decay (6.12). We are going to derive the decay using the bound (6.15) and the orthogonality condition (6.6).

First, we reduce the problem to the analysis of the frozen linear equation,

$$
\dot{X}(t)=A_{1} X(t), \quad t \in \mathbb{R},
$$

where $A_{1}$ is the operator $A_{v_{1}, v_{1}}$ defined by (4.8) with $v_{1}=v\left(t_{1}\right)$ and a fixed $t_{1} \in\left[0, t_{*}\right)$. Then we can apply wellknown methods of scattering theory and then estimate the error by the method of majorants.

Note, that even for the frozen equation (6.16), the decay of type (6.12) for all solutions does not hold without the orthogonality condition of type (6.6). Namely, by (5.7) the equation (6.16) admits the secular solutions

$$
X(t)=\sum_{1}^{3} C_{j} \tau_{j}(v)+\sum_{1}^{3} D_{j}\left[\tau_{j}(v) t+\tau_{j+3}(v)\right]
$$

which arise also by differentiation of the soliton (1.5) in the parameters $a$ and $v$ in the moving coordinate $y=x-v_{1} t$. Hence, we have to take into account the orthogonality condition (6.6) in order to avoid the secular solutions. For this purpose we will apply the corresponding symplectic orthogonal projection which kills the "runaway solutions" (6.17).

Remark 6.1. The solution (6.17) lies in the tangent space $\mathcal{T}_{S\left(\sigma_{1}\right)} \mathcal{S}$ with $\sigma_{1}=\left(b_{1}, v_{1}\right)$ (for an arbitrary $b_{1} \in \mathbb{R}$ ) that suggests an unstable character of the nonlinear dynamics along the solitary manifold (cf. Remark 4.1 iii)).

Definition 6.2. i) For $v \in V$, denote by $\boldsymbol{\Pi}_{v}$ the symplectic orthogonal projection of $\mathcal{E}$ onto the tangent space $\mathcal{T}_{S(\sigma)} \mathcal{S}$, and $\mathbf{P}_{v}=\mathbf{I}-\boldsymbol{\Pi}_{v}$.

ii) Denote by $\mathcal{Z}_{v}=\mathbf{P}_{v} \mathcal{E}$ the space symplectic orthogonal to $\mathcal{T}_{S(\sigma)} \mathcal{S}$ with $\sigma=(b, v)$ (for an arbitrary $b \in \mathbb{R}$ ).

Note that by the linearity,

$$
\Pi_{v} Z=\sum \Pi_{j l}(v) \tau_{j}(v) \Omega\left(\tau_{l}(v), Z\right), \quad Z \in \mathcal{E},
$$

with some smooth coefficients $\boldsymbol{\Pi}_{j l}(v)$. Hence, the projector $\boldsymbol{\Pi}_{v}$, in the variable $y=x-b$, does not depend on $b$, and this explains the choice of the subindex in $\boldsymbol{\Pi}_{v}$ and $\mathbf{P}_{v}$.

Now we have the symplectic orthogonal decomposition

$$
\mathcal{E}=\mathcal{T}_{S(\sigma)} \mathcal{S}+\mathcal{Z}_{v}, \quad \sigma=(b, v),
$$

and the symplectic orthogonality (6.6) can be written in the following equivalent forms,

$$
\Pi_{v(t)} Z(t)=0, \quad \mathbf{P}_{v(t)} Z(t)=Z(t), \quad 0 \leq t<t_{*} .
$$

Remark 6.2. The tangent space $\mathcal{T}_{S(\sigma)} \mathcal{S}$ is invariant under the operator $A_{v, v}$ by Lemma $5.2 \mathrm{i}$ ), hence the space $\mathcal{Z}_{v}$ is also invariant by (5.5): $A_{v, v} Z \in \mathcal{Z}_{v}$ for sufficiently smooth $Z \in \mathcal{Z}_{v}$. 
In Sections 12-18 we will prove the following proposition which will be one of the main ingredients for proving (6.12). Let us consider the Cauchy problem for the equation (6.16) with $A=A_{v, v}$ for a fixed $v \in V$. Recall that the parameter $\beta>3 / 2$ is also fixed.

Proposition 6.2. Let the conditions (1.9) and (1.10) hold, $|v| \leq \bar{v}<2 m$, and $X_{0} \in \mathcal{E}$. Then

i) The equation (6.16), with $A=A_{v, v}$, admits the unique solution $e^{A t} X_{0}:=X(t) \in$ $C(\mathbb{R}, \mathcal{E})$ with the initial condition $X(0)=X_{0}$.

ii) For $X_{0} \in \mathcal{Z}_{v} \cap \mathcal{E}_{\beta}$, the solution $X(t)$ has the following decay,

$$
\left\|e^{A t} X_{0}\right\|_{-\beta} \leq \frac{C_{\beta}(\bar{v})}{(1+|t|)^{3 / 2}}\left\|X_{0}\right\|_{\beta}, \quad t \in \mathbb{R}
$$

\section{Frozen Transversal Dynamics}

Now let us fix an arbitrary $t_{1} \in\left[0, t_{*}\right)$, and rewrite the equation (6.14) in a "frozen form"

$$
\dot{Z}(t)=A_{1} Z(t)+\left(A(t)-A_{1}\right) Z(t)+\tilde{N}(t), \quad 0 \leq t<t_{*},
$$

where $A_{1}=A_{v\left(t_{1}\right), v\left(t_{1}\right)}$ and

$$
A(t)-A_{1}=\left(\begin{array}{cccc}
{\left[w(t)-v\left(t_{1}\right)\right] \cdot \nabla} & 0 & 0 & 0 \\
0 & {\left[w(t)-v\left(t_{1}\right)\right] \cdot \nabla} & 0 & 0 \\
0 & 0 & 0 & 0 \\
0 & 0 & \left\langle\nabla\left(\psi_{v(t) j}-\psi_{v\left(t_{1}\right) j}\right), \nabla \rho_{j}\right\rangle & 0
\end{array}\right) .
$$

The next trick is important since it allows us to kill the "bad terms" $\left[w(t)-v\left(t_{1}\right)\right] \cdot \nabla$ in the operator $A(t)-A_{1}$.

Definition 7.1. Let us change the variables $(y, t) \mapsto\left(y_{1}, t\right)=\left(y+d_{1}(t), t\right)$, where

$$
d_{1}(t):=\int_{t_{1}}^{t}\left(w(s)-v\left(t_{1}\right)\right) d s, \quad 0 \leq t \leq t_{1} .
$$

Next define

$$
Z_{1}(t):=\left(\Psi_{1}\left(y_{1}-d_{1}(t), t\right), \Psi_{2}\left(y_{1}-d_{1}(t), t\right), Q(t), P(t)\right) .
$$

Then we obtain the final form of the "frozen equation" for the transversal dynamics

$$
\dot{Z}_{1}(t)=A_{1} Z_{1}(t)+B_{1}(t) Z_{1}(t)+\tilde{N}_{1}(t), \quad 0 \leq t \leq t_{1}
$$

where $\tilde{N}_{1}(t)=\tilde{N}(t)$ expressed in terms of $y=y_{1}-d_{1}(t)$, and

$$
B_{1}(t)=\left(\begin{array}{cccc}
0 & 0 & 0 & 0 \\
0 & 0 & 0 & 0 \\
0 & 0 & 0 & 0 \\
0 & 0 & \left\langle\nabla\left(\psi_{v(t) j}-\psi_{v\left(t_{1}\right) j}\right), \nabla \rho_{j}\right\rangle & 0
\end{array}\right) .
$$

Lemma 7.1. (see [10]) For $\left(\Psi_{1}, \Psi_{2}, Q, P\right) \in \mathcal{E}_{\alpha}$ with any $\alpha \leq \beta$ the following estimate holds:

$$
\left\|\left(\Psi_{1}\left(y_{1}-d_{1}\right), \Psi_{2}\left(y_{1}-d_{1}\right), Q, P\right)\right\|_{\alpha} \leq\left\|\left(\Psi_{1}, \Psi_{2}, Q, P\right)\right\|_{\alpha}\left(1+\left|d_{1}\right|\right)^{|\alpha|}, \quad d_{1} \in \mathbb{R}^{3} .
$$

Corollary 7.1. The following bounds hold for $0 \leq t \leq t_{1}$

$$
\left\|\tilde{N}_{1}(t)\right\|_{\beta} \leq\|Z(t)\|_{-\beta}^{2}\left(1+\left|d_{1}(t)\right|\right)^{\beta}, \quad\left\|B_{1}(t) Z_{1}(t)\right\|_{\beta} \leq C\|Z(t)\|_{-\beta} \int_{t}^{t_{1}}\|Z(\tau)\|_{-\beta}^{2} d \tau .
$$




\section{Integral Inequality}

The equation (7.4) can be written in the integral form:

$$
Z_{1}(t)=e^{A_{1} t} Z_{1}(0)+\int_{0}^{t} e^{A_{1}(t-s)}\left[B_{1} Z_{1}(s)+\tilde{N}_{1}(s)\right] d s, \quad 0 \leq t \leq t_{1}
$$

Now we apply the symplectic orthogonal projection $\mathbf{P}_{1}:=\mathbf{P}_{v\left(t_{1}\right)}$ to both sides of (8.1). The space $\mathcal{Z}_{1}:=\mathbf{P}_{1} \mathcal{E}$ is invariant with respect to $e^{A_{1} t}$ by Proposition 6.2 ii) (cf. also Remark 6.2). Therefore $\mathbf{P}_{1}$ commutes with the group $e^{A_{1} t}$ and applying (6.21) we obtain that

$$
\left\|\mathbf{P}_{1} Z_{1}(t)\right\|_{-\beta} \leq C \frac{\left\|\mathbf{P}_{1} Z_{1}(0)\right\|_{\beta}}{(1+t)^{3 / 2}}+C \int_{0}^{t} \frac{\left\|\mathbf{P}_{1}\left[B_{1} Z_{1}(s)+\tilde{N}_{1}(s)\right]\right\|_{\beta} d s}{(1+|t-s|)^{3 / 2}} .
$$

The operator $\mathbf{P}_{1}=\mathbf{I}-\boldsymbol{\Pi}_{1}$ is continuous in $\mathcal{E}_{\beta}$ by (6.18). Hence, using 7.6 we obtain that

$$
\begin{aligned}
& \left\|\mathbf{P}_{1} Z_{1}(t)\right\|_{-\beta} \leq \frac{C\left(\bar{d}_{1}(0)\right)}{(1+t)^{3 / 2}}\|Z(0)\|_{\beta} \\
+ & C\left(\bar{d}_{1}(t)\right) \int_{0}^{t} \frac{1}{(1+|t-s|)^{3 / 2}}\left[\|Z(s)\|_{-\beta} \int_{s}^{t_{1}}\|Z(\tau)\|_{-\beta}^{2} d \tau+\|Z(s)\|_{-\beta}^{2}\right] d s, \quad 0 \leq t \leq t_{1} .
\end{aligned}
$$

where $\bar{d}_{1}(t):=\sup _{0 \leq s \leq t}\left|d_{1}(s)\right|$.

Definition 8.1. $t_{*}^{\prime}$ is the exit time

$$
t_{*}^{\prime}=\sup \left\{t \in\left[0, t_{*}\right): \bar{d}_{1}(s) \leq 1, \quad 0 \leq s \leq t\right\}
$$

Now (8.2) implies that for $t_{1}<t_{*}^{\prime}$

$$
\begin{aligned}
& \left\|\mathbf{P}_{1} Z_{1}(t)\right\|_{-\beta} \leq \frac{C}{(1+t)^{3 / 2}}\|Z(0)\|_{\beta} \\
& +C_{1} \int_{0}^{t} \frac{1}{(1+|t-s|)^{3 / 2}}\left[\|Z(s)\|_{-\beta} \int_{s}^{t_{1}}\|Z(\tau)\|_{-\beta}^{2} d \tau+\|Z(s)\|_{-\beta}^{2}\right] d s, \quad 0 \leq t \leq t_{1} .
\end{aligned}
$$

\section{$9 \quad$ Symplectic Orthogonality}

Finally, we are going to change $\mathbf{P}_{1} Z_{1}(t)$ by $Z(t)$ in the left hand side of (8.4). We will prove that it is possible using again that $d_{0} \ll 1$ in (2.12). For the justification we reduce further the exit time. First, we introduce the "majorant"

$$
m(t):=\sup _{s \in[0, t]}(1+s)^{3 / 2}\|Z(s)\|_{-\beta}, \quad t \in\left[0, t_{*}\right)
$$

Let us denote by $\varepsilon$ a fixed positive number which we will specify below.

Definition 9.1. $t_{*}^{\prime \prime}$ is the exit time

$$
t_{*}^{\prime \prime}=\sup \left\{t \in\left[0, t_{*}^{\prime}\right): m(s) \leq \varepsilon, \quad 0 \leq s \leq t\right\}
$$

The following important bound (9.3) allows us to change the norm of $\mathbf{P}_{1} Z_{1}(t)$ in the left hand side of (8.4) by the norm of $Z(t)$. 
Lemma 9.1. (cf.[10]) For sufficiently small $\varepsilon>0$, we have for $t_{1}<t_{*}^{\prime \prime}$

$$
\|Z(t)\|_{-\beta} \leq C\left\|\mathbf{P}_{1} Z_{1}(t)\right\|_{-\beta}, \quad 0 \leq t \leq t_{1},
$$

where $C$ depends only on $\rho$ and $\bar{v}$.

Proof. Since $\left|d_{1}(t)\right| \leq 1$ for $t \leq t_{1}<t_{*}^{\prime \prime}<t_{*}^{\prime}$ then by Lemma 7.1 it suffices to prove that

$$
\left\|Z_{1}(t)\right\|_{-\beta} \leq 2\left\|\mathbf{P}_{1} Z_{1}(t)\right\|_{-\beta}, \quad 0 \leq t \leq t_{1} .
$$

Recall that $\mathbf{P}_{1} Z_{1}(t)=Z_{1}(t)-\boldsymbol{\Pi}_{v\left(t_{1}\right)} Z_{1}(t)$. Then estimate (9.4) will follow from

$$
\left\|\boldsymbol{\Pi}_{v\left(t_{1}\right)} Z_{1}(t)\right\|_{-\beta} \leq \frac{1}{2}\left\|Z_{1}(t)\right\|_{-\beta}, \quad 0 \leq t \leq t_{1} .
$$

Symplectic orthogonality (6.20) implies

$$
\Pi_{v(t), 1} Z_{1}(t)=0, \quad t \in\left[0, t_{1}\right],
$$

where $\Pi_{v(t), 1} Z_{1}(t)$ is $\Pi_{v(t)} Z(t)$ expressed in terms of the variable $y_{1}=y+d_{1}(t)$. Hence, (9.5) follows from (9.6) if the difference $\boldsymbol{\Pi}_{v\left(t_{1}\right)}-\boldsymbol{\Pi}_{v(t), 1}$ is small uniformly in $t$, i.e.

$$
\left\|\boldsymbol{\Pi}_{v\left(t_{1}\right)}-\boldsymbol{\Pi}_{v(t), 1}\right\|<1 / 2, \quad 0 \leq t \leq t_{1} .
$$

It remains to justify (9.7) for small enough $\varepsilon>0$. Formula (6.18) implies the following relation

$$
\Pi_{v(t), 1} Z_{1}(t)=\sum \Pi_{j l}(v(t)) \tau_{j, 1}(v(t)) \Omega\left(\tau_{l, 1}(v(t)), Z_{1}(t)\right),
$$

where $\tau_{j, 1}(v(t))$ are the vectors $\tau_{j}(v(t))$ expressed in the variables $y_{1}$. Since $\left|d_{1}(t)\right| \leq 1$ and $\nabla \tau_{j}$ are smooth and fast decaying at infinity functions, Lemma 7.1 implies that

$$
\left\|\tau_{j, 1}(v(t))-\tau_{j}(v(t))\right\|_{\beta} \leq C\left|d_{1}(t)\right|^{\beta}, \quad 0 \leq t \leq t_{1}
$$

for all $j=1,2, \ldots, 6$. Furthermore,

$$
\tau_{j}(v(t))-\tau_{j}\left(v\left(t_{1}\right)\right)=\int_{t}^{t_{1}} \dot{v}(s) \cdot \nabla_{v} \tau_{j}(v(s)) d s,
$$

and therefore

$$
\left\|\tau_{j}(v(t))-\tau_{j}\left(v\left(t_{1}\right)\right)\right\|_{\beta} \leq C \int_{t}^{t_{1}}|\dot{v}(s)| d s, \quad 0 \leq t \leq t_{1} .
$$

Similarly,

$$
\Pi_{j l}(v(t))-\Pi_{j l}\left(v\left(t_{1}\right)\right)|=| \int_{t}^{t_{1}} \dot{v}(s) \cdot \nabla_{v} \Pi_{j l}(v(s)) d s\left|\leq C \int_{t}^{t_{1}}\right| \dot{v}(s) \mid d s, \quad 0 \leq t \leq t_{1},
$$

since $\left|\nabla_{v} \boldsymbol{\Pi}_{j l}(v(s))\right|$ is uniformly bounded by (6.8). Hence, the bounds (9.7) will follow from (6.18), (9.8) and (9.9)-(9.11) if we establish that $\left|d_{1}(t)\right|$ and the integral in the right hand side of (9.10) can be made as small as we please by choosing $\varepsilon>0$ small enough.

To estimate $d_{1}(t)$, we note that

$$
w(s)-v\left(t_{1}\right)=w(s)-v(s)+v(s)-v\left(t_{1}\right)=\dot{c}(s)+\int_{s}^{t_{1}} \dot{v}(\tau) d \tau
$$


by (6.9). Hence, (7.2), Lemma 6.1 and the definition (9.1) imply that

$$
\begin{aligned}
& \left|d_{1}(t)\right|=\left|\int_{t_{1}}^{t}\left(w(s)-v\left(t_{1}\right)\right) d s\right| \leq \int_{t}^{t_{1}}\left(|\dot{c}(s)|+\int_{s}^{t_{1}}|\dot{v}(\tau)| d \tau\right) d s \\
& \quad \leq C m^{2}\left(t_{1}\right) \int_{t}^{t_{1}}\left(\frac{1}{(1+s)^{3}}+\int_{s}^{t_{1}} \frac{d \tau}{(1+\tau)^{3}}\right) d s \leq C m^{2}\left(t_{1}\right) \leq C \varepsilon^{2}, \quad 0 \leq t \leq t_{1}
\end{aligned}
$$

since $t_{1}<t_{*}^{\prime \prime}$. Similarly,

$$
\int_{t}^{t_{1}}|\dot{v}(s)| d s \leq C m^{2}\left(t_{1}\right) \int_{t}^{t_{1}} \frac{d s}{(1+s)^{3}} \leq C \varepsilon^{2}, \quad 0 \leq t \leq t_{1} .
$$

\section{Decay of Transversal Component}

Here we prove Proposition 6.1.

Step i) We fix $\varepsilon>0$ and $t_{*}^{\prime \prime}=t_{*}^{\prime \prime}(\varepsilon)$ for which Lemma 9.1 holds. Then the bound of type (8.4) holds with $\left\|\mathbf{P}_{1} Z_{1}(t)\right\|_{-\beta}$ in the left hand side replaced by $\|Z(t)\|_{-\beta}$ :

$$
\begin{aligned}
& \|Z(t)\|_{-\beta} \leq \frac{C}{(1+t)^{3 / 2}}\|Z(0)\|_{\beta} \\
& +C \int_{0}^{t} \frac{1}{(1+|t-s|)^{3 / 2}}\left[\|Z(s)\|_{-\beta} \int_{s}^{t_{1}}\|Z(\tau)\|_{-\beta}^{2} d \tau+\|Z(s)\|_{-\beta}^{2}\right] d s, \quad 0 \leq t \leq t_{1}
\end{aligned}
$$

for $t_{1}<t_{*}^{\prime}$. This implies an integral inequality for the majorant $m(t)$ defined in (9.1). Namely, multiplying both sides of $(10.1)$ by $(1+t)^{3 / 2}$, and taking the supremum in $t \in\left[0, t_{1}\right]$, we get

$$
m\left(t_{1}\right) \leq C\|Z(0)\|_{\beta}+C \sup _{t \in\left[0, t_{1}\right]} \int_{0}^{t} \frac{(1+t)^{3 / 2}}{(1+|t-s|)^{3 / 2}}\left[\frac{m(s)}{(1+s)^{3 / 2}} \int_{s}^{t_{1}} \frac{m^{2}(\tau) d \tau}{(1+\tau)^{3}}+\frac{m^{2}(s)}{(1+s)^{3}}\right] d s
$$

for $t_{1} \leq t_{*}^{\prime \prime}$. Taking into account that $m(t)$ is a monotone increasing function, we get

$$
m\left(t_{1}\right) \leq C\|Z(0)\|_{\beta}+C\left[m^{3}\left(t_{1}\right)+m^{2}\left(t_{1}\right)\right] I\left(t_{1}\right), \quad t_{1} \leq t_{*}^{\prime \prime} .
$$

where

$$
I\left(t_{1}\right)=\sup _{t \in\left[0, t_{1}\right]} \int_{0}^{t} \frac{(1+t)^{3 / 2}}{(1+|t-s|)^{3 / 2}}\left[\frac{1}{(1+s)^{3 / 2}} \int_{s}^{t_{1}} \frac{d \tau}{(1+\tau)^{3}}+\frac{1}{(1+s)^{3}}\right] d s \leq \bar{I}<\infty .
$$

Therefore, (10.2) becomes

$$
m\left(t_{1}\right) \leq C\|Z(0)\|_{\beta}+C \bar{I}\left[m^{3}\left(t_{1}\right)+m^{2}\left(t_{1}\right)\right], \quad t_{1}<t_{*}^{\prime \prime} .
$$

This inequality implies that $m\left(t_{1}\right)$ is bounded for $t_{1}<t_{*}^{\prime \prime}$, and moreover,

$$
m\left(t_{1}\right) \leq C_{1}\|Z(0)\|_{\beta}, \quad t_{1}<t_{*}^{\prime \prime},
$$

since $m(0)=\|Z(0)\|_{\beta}$ is sufficiently small by (3.8).

Step ii) The constant $C_{1}$ in the estimate (10.4) does not depend on $t_{*}, t_{*}^{\prime}$ and $t_{*}^{\prime \prime}$ by Lemma 
9.1. We choose $d_{0}$ in (2.12) so small that $\|Z(0)\|_{\beta}<\varepsilon /\left(2 C_{1}\right)$. It is possible due to (3.8). Then the estimate (10.4) implies that $t_{*}^{\prime \prime}=t_{*}^{\prime}$ and therefore (10.4) holds for all $t_{1}<t_{*}^{\prime}$. Then the bound (9.13) holds for all $t<t_{*}^{\prime}$. We choose $\varepsilon$ so small that the right hand side in (9.13) does not exceed one. Then $t_{*}^{\prime}=t_{*}$. Therefore, (10.4) holds for all $t_{1}<t_{*}$, hence the first inequality in (6.3) also holds if $\|Z(0)\|_{\beta}$ is sufficiently small by (9.1) and (9.14). Finally, this implies that $t_{*}=\infty$, hence also $t_{*}^{\prime \prime}=t_{*}^{\prime}=\infty$ and (10.4) holds for all $t_{1}>0$ if $d_{0}$ is small enough.

\section{Soliton Asymptotics}

Here we prove our main Theorem 2.1 under the assumption that the decay (6.12) holds. First we will prove the asymptotics (1.8) for the vector components, and afterwards the asymptotics (1.1) for the fields.

Asymptotics for the vector components From (4.3) we have $\dot{q}=\dot{b}+\dot{Q}$, and from (6.14), (6.15), (4.8) it follows that $\dot{Q}=P+\mathcal{O}\left(\|Z\|_{-\beta}^{2}\right)$. Thus,

$$
\dot{q}=\dot{b}+\dot{Q}=v(t)+\dot{c}(t)+P(t)+\mathcal{O}\left(\|Z\|_{-\beta}^{2}\right) .
$$

The equation (6.10) and the estimates (6.11), (6.12) imply that

$$
|\dot{c}(t)|+|\dot{v}(t)| \leq \frac{C_{1}\left(\rho, \bar{v}, d_{0}\right)}{(1+t)^{3}}, \quad t \geq 0
$$

Therefore, $c(t)=c_{+}+\mathcal{O}\left(t^{-2}\right)$ and $v(t)=v_{+}+\mathcal{O}\left(t^{-2}\right), t \rightarrow \infty$. Since $|P| \leq\|Z\|_{-\beta}$, the estimate (6.12), and (11.2), (11.1) imply that

$$
\dot{q}(t)=v_{+}+\mathcal{O}\left(t^{-3 / 2}\right) .
$$

Similarly,

$$
b(t)=c(t)+\int_{0}^{t} v(s) d s=v_{+} t+a_{+}+\mathcal{O}\left(t^{-1}\right),
$$

hence the second part of (1.8) follows:

$$
q(t)=b(t)+Q(t)=v_{+} t+a_{+}+\mathcal{O}\left(t^{-1}\right)
$$

since $Q(t)=\mathcal{O}\left(t^{-3 / 2}\right)$ by $(6.12)$.

Asymptotics for the fields We apply the approach developed in [12], see also [10]. For the field part of the solution, $\psi(x, t)=\psi_{1}(x, t)+i \psi_{2}(x, t)$ let us define the accompanying soliton field as $\psi_{\mathrm{v}(\mathrm{t})}(x-q(t))$, where we define now $\mathrm{v}(t)=\dot{q}(t)$, cf. (11.1). Then for the difference $z(x, t)=\psi(x, t)-\psi_{\mathrm{v}(\mathrm{t})}(x-q(t))$ we obtain the equation

$$
i \dot{z}(x, t)=\left(-\Delta+m^{2}\right) z(x, t)-i \dot{\mathrm{v}} \cdot \nabla_{\mathrm{v}} \psi_{\mathrm{v}(t)}(x-q(t)) .
$$

Then

$$
z(t)=W_{0}(t) z(0)-\int_{0}^{t} W_{0}(t-s)\left[i \dot{\mathrm{v}}(s) \cdot \nabla_{\mathrm{v}} \psi_{\mathrm{v}(s)}(\cdot-q(s))\right] d s .
$$

To obtain the asymptotics (2.14) it suffices to prove that $z(t)=W_{0}(t) \boldsymbol{\psi}_{+}+r_{+}(t)$ with some $\boldsymbol{\psi}_{+} \in H^{1}$ and $\left\|r_{+}(t)\right\|_{H^{1}}=\mathcal{O}\left(t^{-1 / 2}\right)$. This is equivalent to

$$
W_{0}(-t) z(t)=\boldsymbol{\psi}_{+}+r_{+}^{\prime}(t)
$$


where $\left\|r_{+}^{\prime}(t)\right\|_{H^{1}}=\mathcal{O}\left(t^{-1 / 2}\right)$ since $W^{0}(t)$ is a unitary group in the Sobolev space $\mathcal{F}$ by the energy conservation for the free Schrödinger equation. Finally, (11.7) holds since (11.6) implies that

$$
W_{0}(-t) z(t)=z(0)-\int_{0}^{t} W_{0}(-s) f(s) d s, \quad f(s)=i \dot{\mathrm{v}}(s) \cdot \nabla_{\mathrm{v}} \psi_{\mathrm{v}(s)}(\cdot-q(s)),
$$

where the integral in the right hand side of converges in the Hilbert space $\mathcal{F}$ with the rate $\mathcal{O}\left(t^{-1 / 2}\right)$. The latter holds since $\left\|W_{0}(-s) f(s)\right\|_{H^{1}}=\mathcal{O}\left(s^{-3 / 2}\right)$ by the unitarity of $W_{0}(-s)$ and the decay rate $\|f(s)\|_{H^{1}}=\mathcal{O}\left(s^{-3 / 2}\right)$. Let us prove this rate of decay. It suffices to prove that $|\dot{\mathrm{v}}(s)|=\mathcal{O}\left(s^{-3 / 2}\right)$, or equivalently $|\dot{p}(s)|=\mathcal{O}\left(s^{-3 / 2}\right)$. Substitute (4.2) to the last equation of (1.2) and obtain

$$
\begin{gathered}
\dot{p}(t)=\int\left[\psi_{v(t) j}(x-b(t))+\Psi_{j}(x-b(t), t)\right] \nabla \rho_{j}(x-b(t)-Q(t)) d x=\int \psi_{v(t) j}(y) \nabla \rho_{j}(y) d y \\
+\int \psi_{v(t) j}(y)\left[\nabla \rho_{j}(y-Q(t))-\nabla \rho_{j}(y)\right] d y+\int \Psi_{j}(y, t) \nabla \rho_{j}(y-Q(t)) d y .
\end{gathered}
$$

The first integral in the right hand side is zero by the stationary equations (2.7). The second integral is $\mathcal{O}\left(t^{-3 / 2}\right)$, since $Q(t)=\mathcal{O}\left(t^{-3 / 2}\right)$, and by the conditions (1.9) on $\rho$. Finally, the third integral is $\mathcal{O}\left(t^{-3 / 2}\right)$ by the estimate (6.12). The proof is complete.

\section{Decay for the Linearized Dynamics}

In remaining sections we prove Proposition 6.2 in order to complete the proof of the main result (Theorem 2.1). Here we discuss our general strategy of the proof of the Proposition. We apply the Fourier-Laplace transform

$$
\tilde{X}(\lambda)=\int_{0}^{\infty} e^{-\lambda t} X(t) d t, \quad \operatorname{Re} \lambda>0
$$

to (6.16). According to Proposition 6.2, we expect that the solution $X(t)$ is bounded in the norm $\|\cdot\|_{-\beta}$. Then the integral (12.1) converges and is analytic for $\operatorname{Re} \lambda>0$. We will write $A$ and $v$ instead of $A_{1}$ and $v_{1}$ in all remaining part of the paper. After the Fourier-Laplace transform (6.16) becomes

$$
\lambda \tilde{X}(\lambda)=A \tilde{X}(\lambda)+X_{0}, \quad \operatorname{Re} \lambda>0 .
$$

Let us stress that (12.2) is equivalent to the Cauchy problem for the functions $X(t) \in$ $C_{b}\left([0, \infty) ; \mathcal{E}_{-\beta}\right)$. Hence the solution $X(t)$ is given by

$$
\tilde{X}(\lambda)=-(A-\lambda)^{-1} X_{0}, \quad \operatorname{Re} \lambda>0
$$

if the resolvent $R(\lambda)=(A-\lambda)^{-1}$ exists for $\operatorname{Re} \lambda>0$.

Let us comment on our following strategy in proving the decay (6.12). First, we will construct the resolvent $R(\lambda)$ for $\operatorname{Re} \lambda>0$ and prove that it is a continuous operator in $\mathcal{E}_{-\beta}$. Then $\tilde{X}(\lambda) \in \mathcal{E}_{-\beta}$ and is an analytic function for $\operatorname{Re} \lambda>0$. Second, we have to justify that there exist a (unique) function $X(t) \in C\left([0, \infty) ; \mathcal{E}_{-\beta}\right)$ satisfying (12.1).

The analyticity of $\tilde{X}(\lambda)$ and Paley-Wiener arguments (see [16]) should provide the existence of a $\mathcal{E}_{-\beta}$ - valued distribution $X(t), t \in \mathbb{R}$, with a support in $[0, \infty)$. Formally,

$$
X(t)=\frac{1}{2 \pi} \int_{\mathbb{R}} e^{i \omega t} \tilde{X}(i \omega+0) d \omega, \quad t \in \mathbb{R} .
$$


However, to check the continuity of $X(t)$ for $t \geq 0$, we need additionally a bound for $\tilde{X}(i \omega+0)$ at large $|\omega|$. Finally, for the time decay of $X(t)$, we need an additional information on the smoothness and decay of $\tilde{X}(i \omega+0)$. More precisely, we should prove that the function $\tilde{X}(i \omega+0)$

i) is smooth outside $\omega=0$ and $\omega= \pm \mu$, where $\mu=\mu(v)>0$,

ii) decays in a certain sense as $|\omega| \rightarrow \infty$.

iii)admits the Puiseux expansion at $\omega= \pm \mu$.

iv) is analytic at $\omega=0$ if $X_{0} \in \mathcal{Z}_{v}:=\mathbf{P}_{v} \mathcal{E}$ and $X_{0} \in \mathcal{E}_{\beta}$.

Then the decay (6.12) would follow from the Fourier-Laplace representation (12.4).

We will check the properties of type i)-iv) only for the last two components $\tilde{Q}(\lambda)$ and $\tilde{P}(\lambda)$ of the vector $\tilde{X}(\lambda)=\left(\tilde{\Psi}_{1}(\lambda), \tilde{\Psi}_{2}(\lambda), \tilde{Q}(\lambda), \tilde{P}(\lambda)\right)$. The properties provide the decay (6.12) for the vector components $Q(t)$ and $P(t)$ of the solution $X(t)$. Then for the field components $\Psi_{1}(x, t)$ and $\Psi_{2}(x, t)$ we will use wellknown properties of free Schrödinger equation.

\section{Constructing the Resolvent}

Here we construct the resolvent as a bounded operator in $\mathcal{E}_{-\beta}$ for $\operatorname{Re} \lambda>0$. We will write $\left(\Psi_{1}(y), \Psi_{2}(y), Q, P\right)$ instead of $\left(\tilde{\Psi}_{1}(y, \lambda), \tilde{\Psi}_{2}(y, \lambda), \tilde{Q}(\lambda), \tilde{P}(\lambda)\right)$ to simplify the notations. Then (12.2) reads

$$
(A-\lambda)\left(\begin{array}{c}
\Psi_{1} \\
\Psi_{2} \\
Q \\
P
\end{array}\right)=-\left(\begin{array}{c}
\Psi_{01} \\
\Psi_{02} \\
Q_{0} \\
P_{0}
\end{array}\right)
$$

It is the system of equations

$$
\begin{array}{r|r}
v \cdot \nabla \Psi_{1}(y)-\left(\Delta-m^{2}\right) \Psi_{2}(y)-Q \cdot \nabla \rho_{2}-\lambda \Psi_{1}(y)=-\Psi_{01}(y) & \\
\left(\Delta-m^{2}\right) \Psi_{1}(y)+v \cdot \nabla \Psi_{2}(y)+Q \cdot \nabla \rho_{1}-\lambda \Psi_{2}(y)=-\Psi_{02}(y) & P-\lambda Q=-Q_{0} \\
-\left\langle\nabla \Psi_{j}(y), \rho_{j}(y)\right\rangle+\left\langle\nabla \psi_{v j}(y), Q \cdot \nabla \rho_{j}(y)\right\rangle-\lambda P=-P_{0} & y \in \mathbb{R}^{3} .
\end{array}
$$

Step i) Let us study the first two equations. In Fourier space they become

$$
\begin{aligned}
& -(i k v+\lambda) \hat{\Psi}_{1}(k)+\left(k^{2}+m^{2}\right) \hat{\Psi}_{2}(k)=-\hat{\Psi}_{01}(k)-i Q k \hat{\rho}_{2}, \\
& -\left(k^{2}+m^{2}\right) \hat{\Psi}_{1}(k)-(i k v+\lambda) \hat{\Psi}_{2}(k)=-\hat{\Psi}_{02}(k)+i Q k \hat{\rho}_{1} .
\end{aligned}
$$

Let us invert the matrix of the system and obtain

$$
\left(\begin{array}{cc}
-(i k v+\lambda) & k^{2}+m^{2} \\
-\left(k^{2}+m^{2}\right) & -(i k v+\lambda)
\end{array}\right)^{-1}=\left[(i k v+\lambda)^{2}+\left(k^{2}+m^{2}\right)^{2}\right]^{-1}\left(\begin{array}{cc}
-(i k v+\lambda) & -\left(k^{2}+m^{2}\right) \\
k^{2}+m^{2} & -(i k v+\lambda)
\end{array}\right) .
$$

Taking the inverse Fourier transform we obtain the corresponding fundamental solution

$$
G_{\lambda}(y)=\left(\begin{array}{cc}
v \cdot \nabla-\lambda & \Delta-m^{2} \\
-\Delta+m^{2} & v \cdot \nabla-\lambda
\end{array}\right) g_{\lambda}(y)
$$


where

$$
g_{\lambda}(y)=F_{k \rightarrow y}^{-1} \frac{1}{\left(k^{2}+m^{2}\right)^{2}-(k v-i \lambda)^{2}}=F_{k \rightarrow y}^{-1} \frac{1}{\left(k^{2}+m^{2}-k v+i \lambda\right)\left(k^{2}+m^{2}+k v-i \lambda\right)}
$$

Note that denominator in RHS (13.4) does not vanish for $\operatorname{Re} \lambda>0, k \in \mathbb{R}^{3}$. Moreover it does not vanish for $\operatorname{Re} \lambda>0, k \in \mathbb{C}^{3}$ for sufficiently small $|\operatorname{Im} k|$. Therefore $g_{\lambda}(y)$ decays exponentially by the Paley-Wiener arguments. Let us compute the entries of matrix $G_{\lambda}$ explicitly:

$$
\begin{aligned}
G_{\lambda}^{11}(y) & =G_{\lambda}^{22}(y)=F^{-1} \frac{-i k v-\lambda}{\left(k^{2}+m^{2}\right)^{2}-(k v-i \lambda)^{2}} \\
& =F_{k \rightarrow y}^{-1}\left(\frac{1 / 2 i}{k^{2}+m^{2}-k v+i \lambda}-\frac{1 / 2 i}{k^{2}+m^{2}+k v-i \lambda}\right)=\frac{e^{-\varkappa+|y|-i \frac{v}{2} y}}{8 i \pi|y|}-\frac{e^{-\varkappa-|y|+i \frac{v}{2} y}}{8 i \pi|y|} \\
G_{\lambda}^{21}(y) & =-G_{\lambda}^{12}(y)=F^{-1} \frac{k^{2}+m^{2}}{\left(k^{2}+m^{2}\right)^{2}-(k v-i \lambda)^{2}} \\
& =F_{k \rightarrow y}^{-1}\left(\frac{1 / 2}{k^{2}+m^{2}-k v+i \lambda}+\frac{1 / 2}{k^{2}+m^{2}+k v-i \lambda}\right)=\frac{e^{-\varkappa+|y|-i \frac{v}{2} y}}{8 \pi|y|}+\frac{e^{-\varkappa-|y|+i \frac{v}{2} y}}{8 \pi|y|}
\end{aligned}
$$

where

$$
\varkappa_{ \pm}=\sqrt{m^{2}-\frac{v^{2}}{4} \pm i \lambda}, \quad \operatorname{Re} \varkappa_{ \pm}>0
$$

This implies

Lemma 13.1. i) The operator $G_{\lambda}$ with the integral kernel $G_{\lambda}\left(y-y^{\prime}\right)$, is continuous operator $H^{1}\left(\mathbb{R}^{3}\right) \oplus H^{1}\left(R^{3}\right) \rightarrow H^{2}\left(\mathbb{R}^{3}\right) \oplus H^{2}\left(R^{3}\right)$ for $\operatorname{Re} \lambda>0$.

ii) The formulas (13.5) and (13.6) imply that for every fixed $y$, the matrix function $G_{\lambda}(y)$, $\operatorname{Re} \lambda>0$, admits an analytic continuation in $\lambda$ to the Riemann surface of the algebraic function $\sqrt{\mu^{2}+\lambda^{2}}$ with the branching points $\lambda= \pm i \mu$, where $\mu:=m^{2}-\frac{v^{2}}{4}$.

Thus, from (13.2) and (13.3) we obtain the convolution representation

$$
\begin{aligned}
& \Psi_{1}=-G_{\lambda}^{11} * \Psi_{01}-G_{\lambda}^{12} * \Psi_{02}-\left(G_{\lambda}^{12} * \nabla \rho_{1}\right) \cdot Q+\left(G_{\lambda}^{11} * \nabla \rho_{2}\right) \cdot Q \\
& \Psi_{2}=G_{\lambda}^{12} * \Psi_{01}-G_{\lambda}^{11} * \Psi_{02}-\left(G_{\lambda}^{11} * \nabla \rho_{1}\right) \cdot Q-\left(G_{\lambda}^{12} * \nabla \rho_{2}\right) \cdot Q
\end{aligned}
$$

Step ii) Now we proceed to the last two equations (13.1):

$$
-\lambda Q+P=-Q_{0}, \quad\left\langle\nabla \psi_{v j}, Q \cdot \nabla \rho_{j}\right\rangle-\left\langle\nabla \Psi_{j}, \rho_{j}\right\rangle-\lambda P=-P_{0} .
$$

Let us rewrite equations (13.7) as $\Psi_{j}=\Psi_{j}(Q)+\Psi_{j}\left(\Psi_{01}, \Psi_{02}\right)$, where

$$
\begin{array}{ll}
\Psi_{1}\left(\Psi_{01}, \Psi_{02}\right)=-G_{\lambda}^{11} * \Psi_{01}-G_{\lambda}^{12} * \Psi_{02}, & \Psi_{1}(Q)=\left(-G_{\lambda}^{12} * \nabla \rho_{1}+G_{\lambda}^{11} * \nabla \rho_{2}\right) \cdot Q \\
\Psi_{2}\left(\Psi_{01}, \Psi_{02}\right)=G_{\lambda}^{12} * \Psi_{01}-G_{\lambda}^{11} * \Psi_{02}, & \Psi_{2}(Q)=-\left(G_{\lambda}^{11} * \nabla \rho_{1}+G_{\lambda}^{12} * \nabla \rho_{2}\right) \cdot Q .
\end{array}
$$

Then $\left\langle\nabla \Psi_{j}, \rho_{j}\right\rangle=\left\langle\nabla \Psi_{j}(Q), \rho_{j}\right\rangle+\left\langle\nabla \Psi_{j}\left(\Psi_{01}, \Psi_{02}\right), \rho_{j}\right\rangle$, and the last equation (13.8) becomes

$$
\left\langle\nabla \psi_{v j}, Q \cdot \nabla \rho_{j}\right\rangle-\left\langle\nabla \Psi_{j}(Q), \rho_{j}\right\rangle-\lambda P=-P_{0}+\left\langle\nabla \Psi_{j}\left(\Psi_{01}, \Psi_{02}\right), \rho_{j}\right\rangle=:-P_{0}^{\prime} .
$$

First we compute the term

$$
\left\langle\nabla \psi_{v j}, Q \cdot \nabla \rho_{j}\right\rangle=\sum_{l j}\left\langle\nabla \psi_{v j}, Q_{l} \partial_{l} \rho_{j}\right\rangle=\sum_{l j}\left\langle\nabla \psi_{v j}, \partial_{l} \rho_{j}\right\rangle Q_{l} .
$$


Applying the Fourier transform $F_{y \rightarrow k}$, we have by the Parseval identity and (19.3) that

$$
\begin{aligned}
\sum_{j}\left\langle\partial_{i} \psi_{v j}, \partial_{l} \rho_{j}\right\rangle & =\sum_{j}\left\langle-i k_{i} \hat{\psi}_{v j},-i k_{l} \hat{\rho}_{j}\right\rangle \\
& =\left\langle k_{i} \frac{-\left(k^{2}+m^{2}\right) \hat{\rho}_{1}+i k v \hat{\rho}_{2}}{\left(k^{2}+m^{2}\right)^{2}-(k v)^{2}}, k_{l} \hat{\rho}_{1}\right\rangle+\left\langle k_{i} \frac{-i k v \hat{\rho}_{1}-\left(k^{2}+m^{2}\right) \hat{\rho}_{2}}{\left(k^{2}+m^{2}\right)^{2}-(k v)^{2}}, k_{l} \hat{\rho}_{2}\right\rangle \\
& =-\int \frac{k_{i} k_{l}\left(\left(k^{2}+m^{2}\right)\left(\left|\hat{\rho}_{1}\right|^{2}+\left|\hat{\rho}_{2}\right|^{2}\right)+i(k v)\left(\hat{\rho}_{1} \overline{\hat{\rho}}_{2}-\hat{\rho}_{2} \overline{\hat{\rho}}_{1}\right)\right) d k}{\left(k^{2}+m^{2}\right)^{2}-(k v)^{2}}=:-L_{i l} .
\end{aligned}
$$

As the result, $\left\langle\nabla \psi_{v j}, Q \cdot \nabla \rho_{j}\right\rangle=-L Q$, where $L$ is the $3 \times 3$ matrix with the matrix elements $L_{i l}$. Now let us compute the term $-\left\langle\nabla \Psi_{j}(Q), \rho_{j}\right\rangle=\left\langle\Psi_{j}(Q), \nabla \rho_{j}\right\rangle$. One has

$$
\begin{array}{r}
\left\langle\Psi_{j}(Q), \partial_{i} \rho_{j}\right\rangle=\sum_{l}\left(\left\langle-G_{\lambda}^{12} * \partial_{l} \rho_{1}+G_{\lambda}^{11} * \partial_{l} \rho_{2}, \partial_{i} \rho_{1}\right\rangle-\left\langle G_{\lambda}^{11} * \partial_{l} \rho_{1}+G_{\lambda}^{12} * \partial_{l} \rho_{2}, \partial_{i} \rho_{2}\right\rangle\right) Q_{l} \\
=\sum_{l} H_{i l}(\lambda) Q_{l},
\end{array}
$$

and again by the Parseval identity we have

$$
\begin{aligned}
H_{i l}(\lambda): & =\left\langle-G_{\lambda}^{12} * \partial_{l} \rho_{1}+G_{\lambda}^{11} * \partial_{l} \rho_{2}, \partial_{i} \rho_{1}\right\rangle-\left\langle G_{\lambda}^{11} * \partial_{l} \rho_{1}+G_{\lambda}^{12} * \partial_{l} \rho_{2}, \partial_{i} \rho_{2}\right\rangle \\
& =\left\langle\left[\left(k^{2}+m^{2}\right) \hat{\rho}_{1}-(i k v+\lambda) \hat{\rho}_{2}\right] \hat{g}_{\lambda} k_{l}, k_{i} \hat{\rho}_{1}\right\rangle+\left\langle\left[(i k v+\lambda) \hat{\rho}_{1}+\left(k^{2}+m^{2}\right) \hat{\rho}_{2}\right] \hat{g}_{\lambda} k_{l}, k_{i} \hat{\rho}_{2}\right\rangle \\
& =\int \frac{k_{i} k_{l}\left(\left(k^{2}+m^{2}\right)\left(\left|\hat{\rho}_{1}\right|^{2}+\left|\hat{\rho}_{2}\right|^{2}\right)+(i k v+\lambda)\left(\hat{\rho}_{1} \overline{\hat{\rho}}_{2}-\hat{\rho}_{2} \overline{\hat{\rho}}_{1}\right)\right) d k}{\left(k^{2}+m^{2}\right)^{2}-(k v-i \lambda)^{2}}
\end{aligned}
$$

The matrix $H$ is well defined for $\operatorname{Re} \lambda>0$ since the denominator does not vanish. As the result, $-\left\langle\nabla \Psi_{j}(Q), \rho_{j}\right\rangle=H Q$, where $H$ is the matrix with matrix elements $H_{i l}$. Finally the equations (13.8) become

$$
\mathcal{M}(\lambda)\left(\begin{array}{c}
Q \\
P
\end{array}\right)=\left(\begin{array}{c}
Q_{0} \\
P_{0}^{\prime}
\end{array}\right), \text { where } \mathcal{M}(\lambda)=\left(\begin{array}{cc}
\lambda E & -E \\
L-H(\lambda) & \lambda E
\end{array}\right)
$$

Assume for a moment that the matrix $\mathcal{M}(\lambda)$ is invertible (later we will prove this). Then we obtain

$$
\left(\begin{array}{c}
Q \\
P
\end{array}\right)=\mathcal{M}^{-1}(\lambda)\left(\begin{array}{c}
Q_{0} \\
P_{0}^{\prime}
\end{array}\right), \quad \operatorname{Re} \lambda>0 .
$$

Finally, formula (13.12) and formulas (13.7), where $Q$ is expressed from (13.12), give the expression of the resolvent $R(\lambda)=(A-\lambda)^{-1}, \operatorname{Re} \lambda>0$

Lemma 13.2. The matrix function $\mathcal{M}(\lambda)$ (respectively, $\mathcal{M}^{-1}(\lambda)$ ), $\operatorname{Re} \lambda>0$ admits an analytic (respectively meromorphic) continuation to the Riemann surface of the function $\sqrt{\mu^{2}+\lambda^{2}}, \lambda \in \mathbb{C}$.

Proof. The analytic continuation of $\mathcal{M}(\lambda)$, exists by Lemma 13.1 ii) and the convolution expressions in (13.10) by (1.9). The inverse matrix is then meromorphic since it exists for large $\operatorname{Re} \lambda$. The latter follows from (13.11) since $H(\lambda) \rightarrow 0, \operatorname{Re} \lambda \rightarrow \infty$, by (13.10). 
Here we prove the following

Proposition 14.1. The operator-valued function $R(\lambda): \mathcal{E} \rightarrow \mathcal{E}$ is analytic for $\operatorname{Re} \lambda>0$.

Proof. It is sufficient to prove that the operator $A-\lambda: \mathcal{E} \rightarrow \mathcal{E}$ has a bounded inverse operator for $\operatorname{Re} \lambda>0$. Let us recall, that $A=A_{v, v}$ where $|v|<2 m$.

Step i) Let us prove that $\operatorname{Ker}(A-\lambda)=0$ for $\operatorname{Re} \lambda>0$. Indeed, let us assume that $X_{\lambda}=\left(\Psi_{\lambda 1}, \Psi_{\lambda 2}, Q_{\lambda}, P_{\lambda}\right) \in \mathcal{E}$ satisfies $(A-\lambda) X_{\lambda}=0$, that is $X_{\lambda}$ is a solution to (13.1) with $\Psi_{01}=\Psi_{02}=0$ and $Q_{0}=P_{0}=0$. We have to prove that $X_{\lambda}=0$.

First let us check that $P_{\lambda}=0$. Indeed, the trajectory $X:=X_{\lambda} e^{\lambda t} \in C(\mathbb{R}, \mathcal{E})$ is the solution to the equation $\dot{X}=A X$ that is (5.1) with $w=v$. Then $\mathcal{H}_{v, v}(X(t))$ grows exponentially by (5.8). This growth contradicts to the conservation of $\mathcal{H}_{v, v}$, which follows from Lemma $5.1 \mathrm{ii})$ since $X(t) \in C^{1}\left(\mathbb{R}, \mathcal{E}^{+}\right)$. The latter inclusion follows from Lemma 13.1 since $\left(\Psi_{\lambda 1}, \Psi_{\lambda 2}\right)$ satisfies the equations (13.7) with $\Psi_{01}=\Psi_{02}=0$ and $Q=Q_{\lambda}$.

Now $\lambda Q_{\lambda}=P_{\lambda}=0$ by the third equation of (13.1), hence $Q_{\lambda}=0$ since $\lambda \neq 0$. Finally, $\Psi_{\lambda 1}=0, \Psi_{\lambda 2}=0$ by the equations (13.7) with $Q=Q_{\lambda}=0$.

Step ii) Let us represent $A-\lambda=A_{0}+T$, where

$$
A_{0}=\left(\begin{array}{cccc}
v \cdot \nabla-\lambda & -\left(\Delta-m^{2}\right) & 0 & 0 \\
\Delta-m^{2} & v \cdot \nabla-\lambda & 0 & 0 \\
0 & 0 & -\lambda & 0 \\
0 & 0 & 0-\lambda
\end{array}\right), T=\left(\begin{array}{cccc}
0 & 0 & -\cdot \nabla \rho_{2} & 0 \\
0 & 0 & \cdot \nabla \rho_{1} & 0 \\
0 & 0 & E \\
\left\langle\cdot, \nabla \rho_{1}\right\rangle & \left\langle\cdot, \nabla \rho_{1}\right\rangle & \left\langle\nabla \psi_{v j}, \nabla \rho_{j}\right\rangle & 0
\end{array}\right) .
$$

The operator $T$ is finite-dimensional, and the operator $A_{0}^{-1}$ is bounded in $\mathcal{E}$ by Lemma 13.1. Finally, $A-\lambda=A_{0}\left(I+A_{0}^{-1} T\right)$, where $A_{0}^{-1} T$ is a compact operator. Since we know that $\operatorname{Ker}\left(I+A_{0}^{-1} T\right)=0$, the operator $\left(I+A_{0}^{-1} T\right)$ is invertible by Fredholm theory.

Corollary 14.1. The matrix $\mathcal{M}(\lambda)$ of (13.11) is invertible for $\operatorname{Re} \lambda>0$.

\section{Regularity on the Imaginary Axis}

First, let us describe the continuous spectrum of the operator $A=A_{v, v}$ on the imaginary axis. By definition, the continuous spectrum corresponds to $\omega \in \mathbb{R}$, such that the resolvent $R(i \omega+0)$ is not a bounded operator in $\mathcal{E}$. By the formulas (13.7), this is the case when the Green function $G_{\lambda}\left(y-y^{\prime}\right)$ loses the exponential decay. Thus, $i \omega$ belongs to the continuous spectrum if

$$
|\omega| \geq \mu=m^{2}-v^{2} / 4
$$

By Lemma 13.2, the limit matrix

$$
\mathcal{M}(i \omega):=\mathcal{M}(i \omega+0)=\left(\begin{array}{cc}
i \omega E & -E \\
L-H(i \omega+0) & i \omega E
\end{array}\right), \quad \omega \in \mathbb{R}
$$

exists, and its entries are continuous functions of $\omega \in \mathbb{R}$, smooth for $|\omega|<\mu$ and $|\omega|>\mu$. Recall that the point $\lambda=0$ belongs to the discrete spectrum of the operator $A$ by Lemma $5.2 \mathrm{i}$ ), hence $\mathcal{M}(i \omega+0)$ (probably) also is not invertible at $\omega=0$.

Proposition 15.1. Let $\rho$ satisfy the condition(1.9) and the Wiener condition (1.10), and $|v|<2 m$. Then the limit matrix $\mathcal{M}(i \omega+0)$ is invertible for $\omega \neq 0, \omega \in \mathbb{R}$. 
Proof We will consider separately three cases $0<|\omega|<\mu, \omega=\mu$, and $|\omega|>\mu$. We can assume that $v=(|v|, 0,0)$. Let us denote $F(\omega):=-L+H(i \omega+0), M=m^{2}+k^{2}$, $a=\left|\hat{\rho}_{1}\right|^{2}+\left|\hat{\rho}_{2}\right|^{2}, b=i\left(\hat{\rho}_{1} \overline{\hat{\rho}}_{2}-\hat{\rho}_{2} \overline{\hat{\rho}}_{1}\right)$. Then the entries of the matrix $F$ become

$$
\begin{aligned}
F_{i j} & =\int k_{i} k_{j} d k\left[M a\left(\frac{1}{M^{2}-\left(|v| k_{1}+\omega\right)^{2}}-\frac{1}{M^{2}-\left(|v| k_{1}\right)^{2}}\right)\right. \\
& \left.+b\left(\frac{|v| k_{1}+\omega}{M^{2}-\left(|v| k_{1}+\omega\right)^{2}}-\frac{|v| k_{1}}{M^{2}-\left(|v| k_{1}\right)^{2}}\right)\right] \\
& =\int \frac{k_{i} k_{j} d k}{2}\left[a\left(\frac{1}{M-|v| k_{1}-\omega}+\frac{1}{M+|v| k_{1}+\omega}-\frac{1}{M-|v| k_{1}}-\frac{1}{M+|v| k_{1}}\right)\right. \\
& \left.+b\left(\frac{1}{M-|v| k_{1}-\omega}-\frac{1}{M+|v| k_{1}+\omega}-\frac{1}{M-|v| k_{1}}+\frac{1}{M+|v| k_{1}}\right)\right] .
\end{aligned}
$$

Since $a$ is even, and $b$ is odd we obtain that

$$
F_{i j}=\frac{1}{2} \int d k_{2} d k_{3} \int_{0}^{+\infty} k_{i} k_{j} d k_{1}\left[a f_{1}+b f_{2}\right]
$$

where

$$
\begin{aligned}
f_{1}: & =\frac{1}{M-|v| k_{1}-\omega}+\frac{1}{M+|v| k_{1}+\omega}+\frac{1}{M+|v| k_{1}-\omega}+\frac{1}{M-|v| k_{1}+\omega} \\
& -\frac{2}{M-|v| k_{1}}-\frac{2}{M+|v| k_{1}}, \\
f_{2}: & =\frac{1}{M-|v| k_{1}-\omega}-\frac{1}{M+|v| k_{1}+\omega}+\frac{1}{M-|v| k_{1}+\omega}-\frac{1}{M+|v| k_{1}-\omega} \\
& -\frac{2}{M-|v| k_{1}}+\frac{2}{M+|v| k_{1}}
\end{aligned}
$$

Then by (15.1)

$$
\begin{aligned}
\operatorname{det} \mathcal{M}(i \omega) & =\operatorname{det}\left(\begin{array}{cccccc}
i \omega & 0 & 0 & -1 & 0 & 0 \\
0 & i \omega & 0 & 0 & -1 & 0 \\
0 & 0 & i \omega & 0 & 0 & -1 \\
-F_{11} & -F_{12} & -F_{13} & i \omega & 0 & 0 \\
-F_{12} & -F_{22} & -F_{23} & 0 & i \omega & 0 \\
-F_{13} & -F_{23} & -F_{33} & 0 & 0 & i \omega
\end{array}\right) \\
& =-\omega^{6}-\omega^{4} \sum_{j=1}^{3} F_{j j}-\omega^{2} \sum_{i<j}\left(F_{i i} F_{j j}-F_{i j}^{2}\right)-\operatorname{det}\left(\begin{array}{ccc}
F_{11} & F_{12} & F_{13} \\
F_{12} & F_{22} & F_{23} \\
F_{13} & F_{23} & F_{33}
\end{array}\right)
\end{aligned}
$$

since $F_{i j}=F_{j i}$.

I. First, let us consider the case $0<|\omega|<\mu$. Then the invertibility of $\mathcal{M}(i \omega)$ follows from

Lemma 15.1. For $0<|\omega|<\mu$, the matrix $F$ is positive definite.

Proof. First, let us note that all denominators in (15.4) are positive for $|\omega|<\mu=m^{2}-$ $v^{2} / 4,|v|<2 m$. Indeed,

$$
\left(m^{2}+k^{2}\right)^{2}-\left(\omega+|v| k_{1}\right)^{2}=\left((k-v / 2)^{2}+m^{2}-\frac{v^{2}}{4}-\omega\right)\left((k+v / 2)^{2}+m^{2}-\frac{v^{2}}{4}+\omega\right)>0
$$


Second, $f_{1}>f_{2} \geq 0$ if $|v|<2 m$ and $0<|\omega| \leq \mu$. This is proved in Appendix C.

Finally, the Wiener condition implies

$$
a \pm b=\left|\hat{\rho}_{1}(k) \mp i \hat{\rho}_{2}(k)\right|^{2}>0, \quad \forall k \in \mathbb{R}^{3} .
$$

Therefore $a f_{1}+b f_{2}>0$ and (15.3) is the integral of the symmetric nonnegative definite matrix $k \otimes k=\left(k_{i} k_{j}\right)$ with a positive weight. Hence, the matrix $F$ is positive definite.

II. $\omega= \pm \mu$. Let us consider for example $\omega=\mu=m^{2}-\frac{|v|^{2}}{4}$. In this case (13.10) reads:

$$
H_{i j}(i \mu)=\int \frac{k_{i} k_{j}(M a-(k v+\mu) b) d k}{\left(\left(k_{1}-\frac{|v|}{2}\right)^{2}+k_{2}^{2}+k_{3}^{2}\right)\left(\left(k_{1}+\frac{|v|}{2}\right)^{2}+k_{2}^{2}+k_{3}^{2}+2 \mu\right)} .
$$

Now the integrand has a unique singular point. The singularity is integrable, hence $\operatorname{det} \mathcal{M}(i \omega)$ also is negative by the representations (15.5). Hence, the matrix $\mathcal{M}(i \mu)$ is also invertible.

III. $|\omega|>\mu$. Here we apply an other arguments. Now the invertibility of $\mathcal{M}(i \omega)$ follows from (15.5) by the following lemma (cf. [10])

Lemma 15.2. If (1.10) holds and $\omega>\mu(\omega<-\mu)$, then the matrix $\operatorname{Im} F(\omega)$ is negative (positive) definite.

Proof. We consider the case $\omega>\mu$ (the case $\omega<-\mu$ can be treated similarly). Let us calculate the imaginary part of $F_{i j}$. Since $F_{i j}=H_{i j}(i \omega+0)-L_{i j}$ and $L_{i j}$ is real, we will consider only $H_{i j}(i \omega+0)$. For $\varepsilon>0$ we have

$$
\begin{aligned}
H_{i j}(i \omega+\varepsilon) & =\int \frac{k_{i} k_{j}(M a+(k v+\omega-i \varepsilon) b) d k}{M^{2}-(k v+\omega-i \varepsilon)^{2}}=\frac{1}{2} \int \frac{k_{i} k_{j}(a+b) d k}{M-k v-\omega+i \varepsilon} \\
& +\frac{1}{2} \int \frac{k_{i} k_{j}(a-b) d k}{M+k v+\omega-i \varepsilon}=H_{i j}^{1}(i \omega+\varepsilon)+H_{i j}^{2}(i \omega+\varepsilon) .
\end{aligned}
$$

It suffices to consider only the first summand in (15.7), since the second summand is real for $\varepsilon=0$. Consider the denominator

$$
\hat{D}_{\varepsilon}(k)=k^{2}+m^{2}-k v-\omega+i \varepsilon .
$$

Note that $\hat{D}_{0}(k)=0$ on an ellipsoid $T_{\omega}$, where

$$
T_{\omega}=\left\{k:\left|k-\frac{v}{2}\right|=R:=\sqrt{\omega-\mu}\right\},
$$

Then the Plemelj formula for $C^{1}$-functions implies that

$$
\operatorname{Im} H_{i j}^{1}(i \omega+0)=-\frac{\pi}{2} \int_{T_{\omega}} \frac{k_{i} k_{j}(a+b)}{\left|\nabla \hat{D}_{0}(k)\right|} d S,
$$

where $d S$ is the element of the surface area. Hence, the matrix $\operatorname{Im} H^{1}(i \omega+0)$ is negative definite by (15.6).

Now let us prove that the limit matrix $\mathcal{M}(i \omega+0)$ is invertible. Recall that

$$
\mathcal{M}(i \omega+0)=\left(\begin{array}{ll}
i \omega E & -E \\
-F(i \omega+0) & i \omega E
\end{array}\right)
$$


Then the equation

$$
\mathcal{M}(i \omega+0)\left(\begin{array}{c}
Q \\
P
\end{array}\right)=0
$$

becomes

$$
i \omega Q-P=0, \quad-F Q+i \omega P=0 .
$$

Then $\left(F+\omega^{2}\right) Q=0$, which implies $Q=0$ and then $P=0$ since the matrix $\operatorname{Im} F$ is negative definite for $\omega>\mu$. This completes the proofs of the Proposition 15.1.

Corollary 15.1. Proposition 15.1 implies that the matrix $\mathcal{M}^{-1}(i \omega)$ is smooth in $\omega \in \mathbb{R}$ outside three points $\omega=0, \pm \mu$.

\section{Singular Spectral Points}

Let us recall that the formula (13.12) expresses the Fourier-Laplace transforms $\tilde{Q}(\lambda), \tilde{P}(\lambda)$. Hence, the components are given by the Fourier integral

$$
\left(\begin{array}{l}
Q(t) \\
P(t)
\end{array}\right)=\frac{1}{2 \pi} \int e^{i \omega t} \mathcal{M}^{-1}(i \omega+0)\left(\begin{array}{c}
Q_{0} \\
P_{0}^{\prime}
\end{array}\right) d \omega
$$

if it converges in the sense of distributions. The Corollary 15.1 alone is not sufficient for the proof of the convergence and decay of the integral. Namely, we need an additional information about a regularity of the matrix $\mathcal{M}^{-1}(i \omega)$ at its singular points $\omega=0, \pm \mu$, and some bounds at $|\omega| \rightarrow \infty$. We will analyze all the points separately.

I. First we consider the points $\pm \mu$.

Lemma 16.1. The matrix $\mathcal{M}^{-1}(i \omega)$ admits the following Puiseux expansion in a neighborhood of $\pm \mu$ : there exists an $\varepsilon_{ \pm}>0$ s.t.

$$
\mathcal{M}^{-1}(i \omega)=\sum_{k=0}^{\infty} R_{k}^{ \pm}(\omega \mp \mu)^{k / 2}, \quad|\omega \mp \mu|<\varepsilon_{ \pm}, \quad \omega \in \mathbb{R} .
$$

Proof. It suffices to prove a similar expansion for $\mathcal{M}(i \omega)$. Then (16.2) holds also for $\mathcal{M}^{-1}(i \omega)$, since the matrices $\mathcal{M}( \pm i \mu)$ are invertible. The asymptotics for $\mathcal{M}(i \omega)$ holds by the convolution representation (13.10)

$$
H_{i j}(\lambda)=-\left\langle G_{\lambda}^{12} * \partial_{l} \rho_{1}+G_{\lambda}^{11} * \partial_{l} \rho_{2}, \partial_{i} \rho_{1}\right\rangle-\left\langle G_{\lambda}^{11} * \partial_{l} \rho_{1}+G_{\lambda}^{12} * \partial_{l} \rho_{2}, \partial_{i} \rho_{2}\right\rangle .
$$

since $G_{\lambda}^{i j}$ admit the corresponding Puiseux expansions by the formula (13.5).

II. Second, we study the asymptotic behavior of $\mathcal{M}^{-1}(\lambda)$ at infinity. Let us recall that $\mathcal{M}^{-1}(\lambda)$ was originally defined for $\operatorname{Re} \lambda>0$, and admits a meromorphic continuation to the Riemann surface of the function $\sqrt{m^{2}-\frac{v^{2}}{4}+i \lambda}$ (see Lemma 13.2).

Lemma 16.2. There exist a matrix $R_{0}$ and a matrix-function $R_{1}(\omega)$, such that

$$
\mathcal{M}^{-1}(i \omega)=\frac{R_{0}}{\omega}+R_{1}(\omega), \quad|\omega| \geq \mu+1, \quad \omega \in \mathbb{R}
$$

where, for every $k=0,1,2$, .

$$
\left|\partial_{\omega}^{k} R_{1}(\omega)\right| \leq \frac{C_{k}}{|\omega|^{2}}, \quad|\omega| \geq \mu+1, \quad \omega \in \mathbb{R} .
$$


Proof. The structure (15.1) of the matrix $\mathcal{M}(i \omega)$ provides that it suffices to prove the following estimate for the elements of the matrix $H(i \omega):=H(i \omega+0)$ :

$$
\left|\partial_{\omega}^{k} H_{j j}(i \omega)\right| \leq \frac{C_{k}}{|\omega|}, \quad \omega \in \mathbb{R}, \quad|\omega| \geq \mu+1, \quad j=1,2,3 .
$$

Note, that

$$
G_{\lambda}^{11} * f=\frac{1}{2 i}\left(D_{1}^{-1}(\lambda) f-D_{2}^{-1}(\lambda) f\right), \quad G_{\lambda}^{12} * f=\frac{1}{2}\left(D_{1}^{-1}(\lambda) f+D_{2}^{-1}(\lambda) f\right),
$$

where

$$
D_{1}(\lambda)=-\Delta+m^{2}-i v \cdot \nabla+i \lambda, \quad D_{2}(\lambda)=-\Delta+m^{2}+i v \cdot \nabla-i \lambda, \operatorname{Re} \lambda>0,
$$

and $D_{j}^{-1}(\lambda), j=1,2$ are bounded operators in $L^{2}\left(\mathbb{R}^{3}\right)$. The estimate (16.5) immediately follows from a more general bound

$$
\left\|\partial_{\omega}^{k} D_{j}^{-1}(i \omega+0) f\right\|_{L_{-\sigma}^{2}} \leq \frac{C_{k}(R)}{|\omega|}\|f\|_{L_{\sigma}^{2}}, \quad \omega \in \mathbb{R}, \quad|\omega| \geq \mu+1
$$

which holds for $\sigma>3 / 2$. Namely, (16.5) follows by (1.9) from (16.6) applied to the functions $f(y)=\partial_{l} \rho_{j}(y) \in L_{\sigma}^{2}$.

The bound (16.6) is proved in [1, the bound (A.2')] (see also [15, Thm 8.1]).

III. Finally, we consider the point $\omega=0$ which is most singular. The point is an isolated pole of a finite degree by Lemma 13.2, hence the Laurent expansion holds,

$$
\mathcal{M}^{-1}(i \omega)=\sum_{k=0}^{n} M_{k} \omega^{-k-1}+\mathcal{H}(\omega), \quad|\omega|<\varepsilon_{0},
$$

where $M_{k}$ are $6 \times 6$ complex matrices, $\varepsilon_{0}>0$, and $\mathcal{H}(\omega)$ is an analytic matrix-valued function for complex $\omega$ with $|\omega|<\varepsilon_{0}$.

\section{Time Decay of the Vector Components}

Here we prove the decay (6.12) for the components $Q(t)$ and $P(t)$.

Lemma 17.1. (cf. [10]) Let $X_{0} \in \mathcal{Z}_{v, \beta}$. Then $Q(t), P(t)$ are continuous and

$$
|Q(t)|+|P(t)| \leq \frac{C\left(\rho, \bar{v}, d_{0}\right)}{(1+|t|)^{3 / 2}}, \quad t \geq 0
$$

Proof. The expansions (16.2), (16.4) and (16.7) imply the convergence of the Fourier integral (16.1) in the sense of distributions to a continuous function of $t \geq 0$. Let us prove (17.1). First let us note that the condition $X_{0} \in \mathcal{Z}_{v, \beta}$ implies that the whole trajectory $X(t)$ lies in $\mathcal{Z}_{v, \beta}$. This follows from the invariance of the space $\mathcal{Z}_{v, \beta}$ under the generator $A_{v, v}$ (cf. Remark 6.2). Note that for $X_{0}$ not belonging to $\mathcal{Z}_{v, \beta}$ the components $Q(t)$ and $P(t)$ may contain non-decaying terms which correspond to the singular point $\omega=0$. Indeed, we know that the linearized dynamics admits the secular solutions without decay, see (6.17). The formulas (3.2) give the corresponding components $Q_{S}(t)$ and $P_{S}(t)$ of the secular solutions,

$$
\left(\begin{array}{c}
Q_{S}(t) \\
P_{S}(t)
\end{array}\right)=\sum_{1}^{3} C_{j}\left(\begin{array}{c}
e_{j} \\
0
\end{array}\right)+\sum_{1}^{3} D_{j}\left[\left(\begin{array}{c}
e_{j} \\
0
\end{array}\right) t+\left(\begin{array}{c}
0 \\
e_{j}
\end{array}\right)\right] .
$$


We will show that the symplectic orthogonality condition leads to (17.1). Let us split the Fourier integral (16.1) into three terms using the partition of unity $\zeta_{1}(\omega)+\zeta_{2}(\omega)+\zeta_{3}(\omega)=$ $1, \omega \in \mathbb{R}:$

$$
\begin{aligned}
\left(\begin{array}{l}
Q(t) \\
P(t)
\end{array}\right) & =\frac{1}{2 \pi} \int e^{i \omega t}\left(\zeta_{1}(\omega)+\zeta_{2}(\omega)+\zeta_{3}(\omega)\right) \mathcal{M}^{-1}(i \omega+0)\left(\begin{array}{c}
Q_{0} \\
P_{0}^{\prime}
\end{array}\right) d \omega \\
& =I_{1}(t)+I_{2}(t)+I_{3}(t),
\end{aligned}
$$

where the functions $\zeta_{k}(\omega) \in C^{\infty}(\mathbb{R})$ are supported by

$$
\begin{array}{ll}
\operatorname{supp} \zeta_{1} & \subset\left\{\omega \in \mathbb{R}: \varepsilon_{0} / 2<|\omega|<\mu+2\right\} \\
\operatorname{supp} \zeta_{2} & \subset\{\omega \in \mathbb{R}:|\omega|>\mu+1\} \\
\operatorname{supp} \zeta_{3} & \subset\left\{\omega \in \mathbb{R}:|\omega|<\varepsilon_{0}\right\}
\end{array}
$$

Then

i) The function $I_{1}(t)$ decays, like $(1+|t|)^{-3 / 2}$, by the Puiseux expansion (16.2).

ii) The function $I_{2}(t)$ decays faster than any power of $t$ due to Proposition 16.2.

iii) Finally, the function $I_{3}(t)$ generally does not decay if $n \geq 0$ in the Laurent expansion (16.7). Namely, the contribution of the analytic function $\mathcal{H}(\omega)$ decays faster than any power of $t$. On the other hand, the contribution of the Lorent series,

$$
\left(\begin{array}{c}
Q_{L}(t) \\
P_{L}(t)
\end{array}\right):=\frac{1}{2 \pi} \int e^{i \omega t} \zeta_{3}(\omega) \sum_{k=0}^{n} M_{k}(\omega-i 0)^{-k-1}\left(\begin{array}{c}
Q_{0} \\
P_{0}^{\prime}
\end{array}\right) d \omega, \quad t \in \mathbb{R}
$$

is a polynomial function of $t \in \mathbb{R}$, of a degree $\leq n$, modulo functions decaying faster than any power of $t$. Let us note that the formula (17.2) gives an example of the polynomial functions appeared from (17.5).

We have to show that the symplectic orthogonality condition eliminates the polynomial functions. Our main difficulty is that we do not know anything about the order $n$ of the pole and the Lorent coefficients $M_{k}$ of the matrix $\mathcal{M}^{-1}(i \omega)$ at $\omega=0$.

Our crucial observation is the following:

a) The components (17.2), of the secular solutions, form a linear space $\mathcal{L}_{S}$ of the dimension $\operatorname{dim} \mathcal{L}_{S}=6$.

b) The polynomial functions from (17.5) belong to a linear space $\mathcal{L}_{L}$ of the dimension $\operatorname{dim} \mathcal{L}_{L} \leq 6$, since $\left(Q_{0}, P_{0}^{\prime}\right) \in \mathbb{R}^{6}$.

c) $\mathcal{L}_{S} \subset \mathcal{L}_{L}$ since all the functions (17.2) admits the representation (17.5). The latter follows from the fact that the secular solutions (6.17) can be reproduced by our calculations with the Laplace transform.

Therefore, we conclude that

$$
\mathcal{L}_{L}=\mathcal{L}_{S}
$$

It remains to note that the secular solutions are forbidden since $X_{0} \in \mathcal{Z}_{v, \beta}$. Hence, the polynomial terms in (17.5) vanish that implies the decay (17.1).

More precisely, we know that $X(t)=\mathbf{P}_{v} X(t)$ for all $t \in \mathbb{R}$. On the other hand, the identity (17.6) implies that $X(t)$ can be corrected by a secular solution $X_{S}(t)$ s.t. the corresponding components $Q_{\Delta}(t)$ and $P_{\Delta}(t)$, of the difference $\Delta(t):=X(t)-X_{S}(t)$, decay. Hence, the components $Q(t)$ and $P(t)$, of $X(t)=\mathbf{P}_{v} X(t)=\mathbf{P}_{v}\left[X(t)-X_{S}(t)\right]$, also decay. 


\section{Time Decay of Fields}

Here we prove the decay of the field components $\Psi_{1}(x, t), \Psi_{2}(x, t)$ corresponding to (6.12). The first two equations of (6.16) may be written as one equation:

$$
i \dot{\Psi}(t)=\left(-\Delta+m^{2}+i v \cdot \nabla\right) \Psi-Q(t) \cdot \nabla \rho,
$$

where $\left.\Psi(t)=\Psi_{1}(\cdot, t)+i \Psi_{2}(\cdot, t)\right)$. By Lemma 17.1 we know that $Q$ is continuous function of $t \geq 0$, and

$$
|Q(t)| \leq \frac{C\left(\rho, \bar{v}, d_{0}\right)}{(1+|t|)^{3 / 2}}, \quad t \geq 0 .
$$

Hence, the Proposition 6.2 is reduced now to the following

Proposition 18.1. i) Let a function $Q(t) \in C\left([0, \infty) ; \mathbb{R}^{3}\right)$, and $\Psi_{0} \in H_{\beta}^{1}$. Then the equation (18.1) admits a unique solution $\Psi(t) \in C\left([0, \infty) ; H_{\beta}^{1}\right)$ with the initial condition $\Psi(0)=\Psi_{0}$.

ii) If $\Psi_{0} \in H_{\beta}^{1}$ and the decay (18.2) holds, the corresponding fields also decay uniformly in $v$ :

$$
\|\Psi(t)\|_{1,-\beta} \leq \frac{C\left(\rho, \bar{v}, d_{0},\left\|\Psi_{0}\right\|_{1, \beta}\right)}{(1+|t|)^{3 / 2}}, \quad t \geq 0
$$

for $|v| \leq \bar{v}$ with any $\bar{v} \in(0,2 m)$.

Proof. The statements follow from the Duhamel representation

$$
\Psi(t)=W(t) \Psi_{0}-\int_{0}^{t} W(t-s) Q(s) \cdot \nabla \rho d s, \quad t \geq 0
$$

where $W(t)$ is the dynamical group (propagator) of the free equation

$$
i \dot{\Psi}(t)=\left(-\Delta+m^{2}+i v \cdot \nabla\right) \Psi(t) .
$$

Lemma 18.1. Let $|v| \leq \bar{v}$ with any $\bar{v} \in(0,2 m)$. Then for $\Psi_{0} \in H_{\beta}^{1}$.

$$
\left\|W(t) \Psi_{0}\right\|_{1,-\beta} \leq C(\bar{v})(1+|t|)^{-3 / 2}\left\|\Psi_{0}\right\|_{1, \beta}, \quad t \geq 0 .
$$

Proof. Note that $W(t) \Psi_{0}=e^{-i\left(m^{2}-|v|^{2} / 4\right) t} e^{i v x / 2} \Phi(t)$, where $\Phi(t)$ is a solution to free Schrödinger Equation

$$
i \dot{\Phi}(t)=-\Delta \Phi(t), \quad \Phi(0)=e^{i v x / 2} \Psi_{0}
$$

It is wellknown that $\Phi(t)$ satisfies the estimate $\|\Phi(t)\|_{1,-\beta} \leq C(1+|t|)^{-3 / 2}\|\Phi(0)\|_{1, \beta}, t \geq 0$ (see for example [15]).

Now (18.3) follows from the condition (18.2), and the Duhamel representation (18.4).

\section{Appendix}

\section{A. Solitary waves}

Let us to check the last equation of (2.7):

$$
0=\int\left(\nabla \psi_{v 1}(y) \rho_{1}(y)+\nabla \psi_{v 2}(y) \rho_{2}(y)\right) d y
$$


Let us transfer to the Fourier representation. Set

$$
\hat{\psi}(k):=(2 \pi)^{-3 / 2} \int e^{i k x} \psi(x) d x
$$

It is easy to compute that

$$
-i k v \hat{\psi}_{v 1}+\left(k^{2}+m^{2}\right) \hat{\psi}_{v 2}=-\hat{\rho}_{2}, \quad\left(k^{2}+m^{2}\right) \hat{\psi}_{v 1}+i k v \hat{\psi}_{v 2}=-\hat{\rho}_{1} .
$$

Therefore

$$
\hat{\psi}_{v 1}(k)=\frac{-\left(k^{2}+m^{2}\right) \hat{\rho}_{1}(k)+i k v \hat{\rho}_{2}(k)}{\left(k^{2}+m^{2}\right)^{2}-(k v)^{2}}, \quad \hat{\psi}_{v 2}(k)=\frac{-i k v \hat{\rho}_{1}(k)-\left(k^{2}+m^{2}\right) \hat{\rho}_{2}(k)}{\left(k^{2}+m^{2}\right)^{2}-(k v)^{2}} .
$$

By Parseval identity (19.1) becames

$$
0=\int k_{j}\left(\hat{\psi}_{v 1} \overline{\hat{\rho}}_{1}+\hat{\psi}_{v 2} \overline{\hat{\rho}}_{2}\right) d k=\int \frac{k_{j}\left[-\left(k^{2}+m^{2}\right)\left(\left|\hat{\rho}_{1}\right|^{2}+\left|\hat{\rho}_{2}\right|^{2}\right)+i k v\left(\hat{\rho}_{2} \overline{\hat{\rho}}_{1}-\hat{\rho}_{1} \overline{\hat{\rho}}_{2}\right)\right] d k}{\left(k^{2}+m^{2}\right)^{2}-(k v)^{2}},
$$

which is true, since the integrand is odd.

\section{B. Computing $\Omega\left(\tau_{i}, \tau_{j}\right)$}

Let us to justify the formulas (3.4)-(3.6) for the matrix $\Omega$. For $j, l=1,2,3$ one has from (3.2) and (3.1)

$$
\begin{gathered}
\Omega\left(\tau_{j}, \tau_{l}\right)=\left\langle\partial_{j} \psi_{v 1}, \partial_{l} \psi_{v 2}\right\rangle-\left\langle\partial_{j} \psi_{v 2}, \partial_{l} \psi_{v 1}\right\rangle \\
\Omega\left(\tau_{j+3}, \tau_{l+3}\right)=\left\langle\partial_{v_{j}} \psi_{v 1}, \partial_{v_{l}} \psi_{v 2}\right\rangle-\left\langle\partial_{v_{j}} \psi_{v 2}, \partial_{v_{l}} \psi_{v 1}\right\rangle,
\end{gathered}
$$

and

$$
\Omega\left(\tau_{j}, \tau_{l+3}\right)=-\left\langle\partial_{j} \psi_{v 1}, \partial_{v_{l}} \psi_{v 2}\right\rangle+\left\langle\partial_{j} \psi_{v 2}, \partial_{v_{l}} \psi_{v 1}\right\rangle+e_{j} \cdot e_{l} .
$$

Differentiating (19.2) we get

$$
\partial_{v_{j}} \hat{\psi}_{v 1}=\frac{k_{j} k v \hat{\psi}_{v 1}-i k_{j}\left(k^{2}+m^{2}\right) \hat{\psi}_{v 2}}{\left(k^{2}+m^{2}\right)^{2}-(k v)^{2}}, \quad \partial_{v_{j}} \hat{\psi}_{v 2}=\frac{i k_{j}\left(k^{2}+m^{2}\right) \hat{\psi}_{v 1}+k_{j} k v \hat{\psi}_{v 2}}{\left(k^{2}+m^{2}\right)^{2}-(k v)^{2}}, j=1,2,3 .
$$

Then for $j, l=1,2,3$ we obtain from (19.4) by the Parseval identity that

$$
\Omega\left(\tau_{j}, \tau_{l}\right)=\int k_{j} k_{l} d k\left(\hat{\psi}_{v 1} \overline{\hat{\psi}}_{v 2}-\hat{\psi}_{v 2} \overline{\hat{\psi}}_{v 1}\right)=0
$$

since the function $\hat{\psi}_{v c}=\hat{\psi}_{v 1} \overline{\hat{\psi}}_{v 2}-\hat{\psi}_{v 2} \overline{\hat{\psi}}_{v 1}$ is odd. Similarly, by (19.5) and (19.7)

$\Omega\left(\tau_{j+3}, \tau_{l+3}\right)=-\int \frac{k_{j} k_{l}\left(2 i\left(k^{2}+m^{2}\right) k v\left(\left|\hat{\psi}_{v 1}\right|^{2}+\left|\hat{\psi}_{v 2}\right|^{2}\right)-\left(\left(k^{2}+m^{2}\right)^{2}+(k v)^{2}\right) \hat{\psi}_{v c}\right) d k}{\left(\left(k^{2}+m^{2}\right)^{2}-(k v)^{2}\right)^{2}}=0$.

Finally, by (19.6),

$$
\Omega\left(\tau_{j}, \tau_{l+3}\right)=\int \frac{k_{j} k_{l}\left(\left(k^{2}+m^{2}\right)\left(\left|\hat{\psi}_{v 1}\right|^{2}+\left|\hat{\psi}_{v 2}\right|^{2}\right)+i k v \hat{\psi}_{v c}\right) d k}{\left(k^{2}+m^{2}\right)^{2}-(k v)^{2}}+e_{j} \cdot e_{l} .
$$

Now (3.4) - (3.6) are proved. 
C. Positivity of $f_{1}$ and $f_{2}$

Here we check the inequalities which we have used in the proof of Lemma 15.1:

$$
\begin{aligned}
& \text { 1) } f_{1}=\left(\frac{1}{M-|v| k_{1}-\omega}+\frac{1}{M-|v| k_{1}+\omega}-\frac{2}{M-|v| k_{1}}\right) \\
& +\left(\frac{1}{M+|v| k_{1}-\omega}+\frac{1}{M+|v| k_{1}+\omega}-\frac{2}{M+|v| k_{1}}\right)>0, \\
& \text { 2) } f_{2}=\left(\frac{1}{M-|v| k_{1}-\omega}+\frac{1}{M-|v| k_{1}+\omega}-\frac{2}{M-|v| k_{1}}\right) \\
& -\left(\frac{1}{M+|v| k_{1}-\omega}+\frac{1}{M+|v| k_{1}+\omega}-\frac{2}{M+|v| k_{1}}\right) \geq 0
\end{aligned}
$$

under the conditions $|v|<2 m, 0<|\omega| \leq \mu=m^{2}-v^{2} / 4$. First, let us note that the expressions in each bracets is positive, since

$$
\frac{1}{b-a}+\frac{1}{b+a}-\frac{2}{b}=\frac{2 a^{2}}{(b+a)(b-a) b}>0
$$

if $b-a, b+a \geq 0, b>0$ and it immediately implies that $f_{1}>0$. Next, the first summand in LHS of (19.11) obviously is not less than the second summand since $|v| k_{1} \geq 0$. Therefore $f_{2} \geq 0$ and $f_{2}<f_{1}$.

\section{References}

[1] S.Agmon, Spectral properties of Schrdinger operators and scattering theory, Ann. Sc. Norm. Super. Pisa, Cl. Sci. Ser. 2 IV(1975), 151-218.

[2] V.S.Buslaev, G.S.Perelman, On nonlinear scattering of states which are close to a soliton, pp. 49-63 in: Méthodes Semi-Classiques, Vol.2 Colloque International (Nantes, juin 1991), Asterisque 208 (1992).

[3] V.S.Buslaev, G.S.Perelman, Scattering for the nonlinear Schrödinger equation: states close to a soliton, St.Petersburg Math. J.4 (1993), 1111-1142.

[4] V.S.Buslaev, G.S.Perelman, On the stability of solitary waves for nonlinear Schrödinger equations, Trans. Amer. Math. Soc. 164, 75-98 (1995).

[5] V.S.Buslaev, C.Sulem, On asymptotic stability of solitary waves for nonlinear Schrödinger equations, Ann. Inst. Henri Poincaré, Anal. Non Linéaire 20 (2003), No.3, 419-475.

[6] S.Cuccagna, Stabilization of solutions to nonlinear Schrödinger equations, Commun. Pure Appl. Math. 54 (2001), No.9, 1110-1145.

[7] M.Grillakis, J.Shatah, W.A.Strauss, Stability theory of solitary waves in the presence of symmetry I, II, J. Func. Anal. 74 (1987), 160-197; 94 (1990), 308-348.

[8] V.Imaikin, A.Komech, P.Markowich, Scattering of solitons of the Klein-Gordon equation coupled to a classical particle, Journal of Mathematical Physics, 44 (2003), no.3, 1202-1217. 
[9] V.Imaikin, A.Komech, N.Mauser, Soliton-type asymptotics for the coupled MaxwellLorentz equations, Ann. Inst. Poincaré, Phys. Theor. 5 (2004), 1117-1135.

[10] V.Imaikin, A.Komech, B.Vainberg, Scattering of solitons in the Klein-Gordon equation coupled to a particle, submitted to Comm. Math. Phys., 2005.

[11] V.Imaikin, A.Komech, H.Spohn, Soliton-like asymptotics and scattering for a particle coupled to Maxwell field, Russian Journal of Mathematical Physics 9 (2002), no.4, 428-436.

[12] V.Imaikin, A.Komech, H.Spohn, Scattering theory for a particle coupled to a scalar field, Journal of Discrete and Continuous Dynamical Systems 10 (2003), no.1\&2, 387-396.

[13] V.Imaikin, A.Komech, H.Spohn, Rotating charge coupled to the Maxwell field: scattering theory and adiabatic limit, Monatsh. Math. 142 (2004), no.1-2, 143-156.

[14] A.Jensen, On a unified approach to resolvent expansions for Schrödinger operators, RIMS Kokyuroku 1208, 91-103 (2001).

[15] A.Jensen, T.Kato, Spectral properties of Schrödinger operators and time-decay of the wave functions, Duke Math. Journal, 46, 583-611 (1979).

[16] A.I.Komech, Linear Partial Differential Equations with Constant Coefficients, p.127260 in: Yu.V.Egorov, A.I.Komech, M.A.Shubin, Elements of the Modern Theory of Partial Differential Equations, Springer, Berlin, 1999.

[17] A.Komech, M.Kunze, H.Spohn, Effective dynamics for a mechanical particle coupled to a wave field, Comm. Math.Phys. 203 (1999), 1-19.

[18] A.Komech, M.Kunze, H.Spohn, Long-time asymptotics for a classical particle interacting with a scalar wave field, Comm. Partial Differential Equations 22 (1997), 307-335.

[19] A.I.Komech, H.Spohn, Soliton-like asymptotics for a classical particle interacting with a scalar wave field, Nonlin. Analysis 33 (1998), 13-24.

[20] J.Miller, M.Weinstein, Asymptotic stability of solitary waves for the regularized longwave equation, Comm. Pure Appl. Math. 49 (1996), no. 4, 399-441.

[21] I.M.Sigal, Nonlinear wave and Schrödinger equations. I. Instability of periodic and quasiperiodic solutions, Comm. Math. Phys. 153 (1993), no. 2, 297-320.

[22] A.Soffer, M.I.Weinstein, Resonances, radiation damping and instability in Hamiltonian nonlinear wave equations, Invent. Math. 136 (1999), no. 1, 9-74.

[23] A.Soffer, M.I.Weinstein, Selection of the ground state for nonlinear Schrödinger equations, preprint ArXiv:nlin.PS/0308020, 2003.

[24] H.Spohn, Dynamics of Charged Particles and Their Radiation Field, Cambridge University Press, Cambridge, 2004.

[25] M.Weinstein, Modulational stability of ground states of nonlinear Schrdinger equations, SIAM J. Math. Anal. 16 (1985), no. 3, 472-491. 